\title{
AN APPROACH FOR WEIGHTED MIXED-NORM ESTIMATES FOR PARABOLIC EQUATIONS WITH LOCAL AND NON-LOCAL TIME DERIVATIVES
}

\author{
HONGJIE DONG AND DOYOON KIM
}

\begin{abstract}
We give a unified approach to weighted mixed-norm estimates and solvability for both the usual and time fractional parabolic equations in nondivergence form when coefficients are merely measurable in the time variable. In the spatial variables, the leading coefficients locally have small mean oscillations. Our results extend the previous result in [6] for unmixed $L_{p}$-estimates without weights.
\end{abstract}

\section{INTRODUCTION}

In this paper, we consider parabolic equations in nondivergence form

$$
-\partial_{t} u+a^{i j}(t, x) D_{i j} u+b^{i}(t, x) D_{i} u+c(t, x) u=f(t, x)
$$

as well as parabolic equations with a non-local type time derivative term of the form

$$
-\partial_{t}^{\alpha} u+a^{i j}(t, x) D_{i j} u+b^{i}(t, x) D_{i} u+c(t, x) u=f(t, x)
$$

in $(0, T) \times \mathbb{R}^{d}$, where $\partial_{t}^{\alpha} u$ is the Caputo fractional derivative of order $\alpha \in(0,1)$. See Section 2 for the definition of $\partial_{t}^{\alpha} u$.

This paper is a continuation of [6], in which we proved that for any given $f \in$ $L_{p}\left((0, T) \times \mathbb{R}^{d}\right)$, there exists a unique solution $u$ to the equation (1.2) in $(0, T) \times \mathbb{R}^{d}$ with the zero initial condition, and $u$ satisfies

$$
\left\|\left|\partial_{t}^{\alpha} u\right|+|u|+|D u|+\left|D^{2} u\right|\right\|_{L_{p}\left((0, T) \times \mathbb{R}^{d}\right)} \leq N\|f\|_{L_{p}\left((0, T) \times \mathbb{R}^{d}\right)}
$$

under the assumptions that the coefficients are bounded and measurable, and $a^{i j}=a^{i j}(t, x)$ satisfy the uniform ellipticity condition and have small (bounded) mean oscillations (small BMO) with respect to the space variables. Such type of coefficients were first introduced by Krylov in [11] for (1.1) and the corresponding divergence form equations in $L_{p}$ spaces. His proof is based on the mean oscillation argument together with the Fefferman-Stein sharp function theorem and the Hardy-Littlewood maximal function theorem. The results in 11] were generalized later in [12] to the mixed-norm $L_{p}\left(L_{q}\right)$ spaces with $p \geq q$, and in [5] to the weighted mixed-norm $L_{p}\left(L_{q}\right)$ spaces with arbitrary $p, q \in(1, \infty)$ and Muckenhoupt weights. In these papers, the mean oscillation argument is used, and in particular, in [5] a version of the Fefferman-Stein sharp function theorem is proved in weighted mixed-norm Lebesgue spaces by using the extrapolation theorem. We note that for

2010 Mathematics Subject Classification. 35R11, 26A33, 35R05.

Key words and phrases. parabolic equation, time fractional derivative, mean oscillation estimates, measurable coefficients.

D. Kim was supported by the National Research Foundation of Korea (NRF) grant funded by the Korea government (MSIT) (2019R1A2C1084683). 
time-dependent equations, such mixed-norm estimates are desirable, for example, when one wants to have better integrability of traces of solutions for each time slice when studying linear or nonlinear equations.

To the best of our knowledge, Equation (1.2) in mixed-norm Lebesgue spaces was first considered in [10] for any $\alpha \in(0,2)$, under the conditions that the leading coefficients $a^{i j}$ are piecewise continuous in time and uniformly continuous in the spatial variables. Very recently, the result in 14 was extended to weighted mixednorm Lebesgue spaces for the fractional heat equation

$$
-\partial_{t}^{\alpha} u+\Delta u=f .
$$

The proofs in [10] and [14] are based upon a representation formula in terms of the fundamental solution to the time fractional heat operator $-\partial_{t}^{\alpha}+\Delta$ together with a perturbation argument using the Fefferman-Stein sharp function theorem and the Hardy-Littlewood maximal function theorem. On the other hand, the proof in 6] (also see [7]) is quite different from those in [10, 14]. It is based upon a modified level set argument used in [2] in order to improve the integrability of solution iteratively. In particular, we did not use the representation formula, which enables us to treat more general operators as those in [6, 7] with coefficients merely measurable in time or in one of the spatial variables. For other results in this direction, we refer the reader to [3, 15, 17] and the references therein.

In view of the results in [5] and [10, 14, it is natural to ask whether the result in 6] can be extended to the mixed-norm $L_{p}\left(L_{q}\right)$ spaces and whether it is possible to also include weights. Unfortunately, it turns out that these extensions cannot be made by using the technique of iteration and the level set argument in [6]. In this paper, we give a definite answer to these two questions. In particular, our main theorem reads that under the same assumptions on the coefficients as in [6], for any $p, q \in(1, \infty)$ and Muckenhoupt weights $w_{1}(t) \in A_{p}(\mathbb{R}), w_{2}(x) \in A_{q}\left(\mathbb{R}^{d}\right)$, if $u$ satisfies (1.2) with the zero initial condition, then it holds that

$$
\left\|\left|\partial_{t}^{\alpha} u\right|+|u|+|D u|+\left|D^{2} u\right|\right\|_{L_{p, q, w}\left((0, T) \times \mathbb{R}^{d}\right)} \leq N\|f\|_{L_{p, q, w}\left((0, T) \times \mathbb{R}^{d}\right)},
$$

where $w=w_{1}(t) w_{2}(x)$ and $N$ is independent of $u$ and $f$. See Section 2 for the definition of the $L_{p, q, w}$-norm. Furthermore, we show that for any $f \in L_{p, q, w}\left((0, T) \times \mathbb{R}^{d}\right)$, there is a unique solution $u$ (in the appropriate weighted mixed-norm Sobolev space) to (1.2) with the zero initial condition.

For the proof, we adapt the mean oscillation argument in [11, 12, 5] mentioned above. For this, we need to establish a Hölder estimate of $D^{2} v$, where $v$ satisfies a certain homogeneous equation with coefficients depending only on $t$. Such an estimate can be obtained relatively easily for parabolic equations with the local time derivative term $u_{t}$ via somewhat standard local estimates. However, if the non-local time derivative is present, the local estimates do not work when improving the regularity of $v$ because the non-local time derivative of $v$ depends on all past states of $v$. To overcome the difficulty from the non-local effect in time, our strategy is to consider an infinite cylinder $\left(-\infty, t_{0}\right) \times B_{r}\left(x_{0}\right)$ instead of the usual parabolic cylinder $Q_{r}\left(t_{0}, x_{0}\right)$ used in the aforementioned papers, and apply the Hardy-Littlewood maximal function for strong maximal functions. We estimate the Hölder semi-norm of $D^{2} v$ by applying a bootstrap argument which relies on the (unmixed) $L_{p}$ estimate and the Sobolev type embedding results obtained in [6]. For $w:=u-v$, which satisfies a nonhomogeneous equation also in the infinite cylinder with the zero Dirichlet boundary condition, we first apply the Poincaré inequality 
to bound $w$ by $f$, and then use a cutoff argument with a sequence of cutoff functions and again use the $L_{p}$ estimate in $\left[6\right.$ in order to estimate $D^{2} w$. This gives a new decomposition of the solution, which works for both (1.1) and (1.2). Since the argument for the usual parabolic equation (1.1) is less involved, even though the solvability result was already obtained in [5, to illustrate the idea in a simple setting we present the proof of the mean oscillation estimate for (1.1) in Section 3. That is, in this paper we not only extend the results in 6 to the weighted mixed-norm case, but also present a new and alternative approach to obtaining the mean oscillation estimates for both (1.1) and (1.2).

The remaining part of the paper is organized as follows. In the next section, we introduce some notation and state the main result of the paper. In Section 3, we show the mean oscillation estimate for (1.1) by using the new decomposition. In Section 4 we derive the corresponding mean oscillation estimate for (1.2). Finally, we complete the proof of the main theorem in Section 5

\section{Notation AND main Results}

We first introduce some notation used throughout the paper. For $\alpha \in(0,1)$ and $S \in \mathbb{R}$, we denote

$$
I_{S}^{\alpha} u=\frac{1}{\Gamma(\alpha)}=\frac{1}{\Gamma(\alpha)} \int_{S}^{t}(t-s)^{\alpha-1} u(s, x) d s .
$$

We write $\partial_{t}^{\alpha} u=\partial_{t} u$ if $\alpha=1$ and

$$
\partial_{t}^{\alpha} u=\frac{1}{\Gamma(1-\alpha)} \int_{0}^{t}(t-s)^{-\alpha} \partial_{t} u(s, x) d s
$$

for $\alpha \in(0,1)$. Note that $\partial_{t}^{\alpha} u=\partial_{t} I_{0}^{\alpha} u$ for a sufficiently smooth $u$ with $u(0, x)=0$. In some places we use $\partial_{t}^{\alpha} u$ to indicate $\partial_{t} I_{S}^{\alpha} u$ for $u(S, x)=0$, where $S \neq 0$.

For $\alpha \in(0,1]$, we denote the parabolic cylinder by

$$
Q_{r_{1}, r_{2}}(t, x)=\left(t-r_{1}^{2 / \alpha}, t\right) \times B_{r_{2}}(x), \quad Q_{r}(t, x)=Q_{r, r}(t, x) .
$$

We often write $B_{r}$ and $Q_{r}$ for $B_{r}(0)$ and $Q_{r}(0,0)$. For $-\infty<S<T<\infty, \Omega \subset \mathbb{R}^{d}$, we denote the parabolic boundary of the cylinder $(S, T) \times \Omega$ by

$$
\partial_{p}((S, T) \times \Omega)=((S, T) \times \partial \Omega) \cup\left\{(t, x) \in \mathbb{R}^{d+1}: t=S, x \in \bar{\Omega}\right\} .
$$

For $p \in(1, \infty)$ and $k \in\{1,2, \ldots\}$, let $A_{p}\left(\mathbb{R}^{k}, d x\right)$ be the set of all non-negative functions $w$ on $\mathbb{R}^{k}$ such that

$$
[w]_{A_{p}}:=\sup _{x_{0} \in \mathbb{R}^{k}, r>0}\left(f_{B_{r}\left(x_{0}\right)} w(x) d x\right)\left(f_{B_{r}\left(x_{0}\right)}(w(x))^{\frac{-1}{p-1}} d x\right)^{p-1}<\infty,
$$

where $B_{r}\left(x_{0}\right)=\left\{x \in \mathbb{R}^{k}:\left|x-x_{0}\right|<r\right\}$. Recall that $[w]_{A_{p}} \geq 1$.

For $w(t, x)=w_{1}(t) w_{2}(x)$, where $(t, x) \in \mathbb{R} \times \mathbb{R}^{d}$, and $w_{1} \in A_{p}(\mathbb{R}, d t), w_{2} \in$ $A_{q}\left(\mathbb{R}^{d}, d x\right)$, we set $L_{p, q, w}\left((0, T) \times \mathbb{R}^{d}\right)$ to be the set of all measurable functions $f$ defined on $(0, T) \times \mathbb{R}^{d}$ satisfying

$$
\left(\int_{0}^{T}\left(\int_{\mathbb{R}^{d}}|f(t, x)|^{p} w_{2}(x) d x\right)^{q / p} w_{1}(t) d t\right)^{1 / q}<\infty .
$$


If $p=q$ and $w=1, L_{p, q, w}\left((0, T) \times \mathbb{R}^{d}\right)$ becomes the usual $L_{p}\left((0, T) \times \mathbb{R}^{d}\right)$. We write $u \in \mathbb{H}_{p, q, w, 0}^{\alpha, 2}\left((0, T) \times \mathbb{R}^{d}\right)$ if there exists a sequence $\left\{u_{n}\right\}$ such that $u_{n} \in$ $C_{0}^{\infty}\left([0, T] \times \mathbb{R}^{d}\right), u_{n}(0, x)=0$, and

$$
\left\|u_{n}-u\right\|_{p, q, w}+\left\|D u_{n}-D u\right\|_{p, q, w}+\left\|D^{2} u_{n}-D^{2} u\right\|_{p, q, w}+\left\|\partial_{t}^{\alpha} u_{n}-\partial_{t}^{\alpha} u\right\|_{p, q, w} \rightarrow 0
$$

as $n \rightarrow \infty$, where $\|\cdot\|_{p, q, w}=\|\cdot\|_{L_{p, q, w}\left((0, T) \times \mathbb{R}^{d}\right)}$. We often write $\mathbb{H}_{p, w, 0}^{\alpha, 2}\left((0, T) \times \mathbb{R}^{d}\right)$ if $p=q$. In particular, we see that if $\alpha=1, p=q$, and $w(t, x)=1$, then

$$
\mathbb{H}_{p, 0}^{1,2}\left((0, T) \times \mathbb{R}^{d}\right)=\left\{u \in W_{p}^{1,2}\left((0, T) \times \mathbb{R}^{d}\right): u(0, x)=0\right\},
$$

where

$$
W_{p}^{1,2}\left((0, T) \times \mathbb{R}^{d}\right)=\left\{u: u, D u, D^{2} u, \partial_{t} u \in L_{p}\left((0, T) \times \mathbb{R}^{d}\right)\right\} .
$$

However, for $\alpha \in(0,1)$, as remarked in [6, Remark 3.4],

$$
\begin{aligned}
\mathbb{H}_{p, 0}^{\alpha, 2}\left((0, T) \times \mathbb{R}^{d}\right) & \subsetneq \mathbb{H}_{p}^{\alpha, 2}\left((0, T) \times \mathbb{R}^{d}\right) \\
& \subsetneq\left\{u: u, D u, D^{2} u, \partial_{t}^{\alpha} u \in L_{p}\left((0, T) \times \mathbb{R}^{d}\right)\right\} .
\end{aligned}
$$

See [6] for more details about $\mathbb{H}_{p}^{\alpha, 2}((0, T) \times \Omega)$ and $\mathbb{H}_{p, 0}^{\alpha, 2}((0, T) \times \Omega), \Omega \subset \mathbb{R}^{d}$. We use the notation $u \in \mathbb{H}_{p, 0, \text { loc }}^{\alpha, 2}\left((0, T) \times \mathbb{R}^{d}\right)$ to indicate that

$$
u \in \mathbb{H}_{p, 0}^{\alpha, 2}\left((0, T) \times B_{R}\right)
$$

for any $R>0$. In particular, when $\alpha=1$, by $u \in W_{p, \text { loc }}^{1,2}\left((-\infty, T) \times \mathbb{R}^{d}\right)$ we mean that $u \in W_{p}^{1,2}\left((-\infty, T) \times B_{R}\right)$ for any $R>0$. Likewise, $f \in L_{p, \text { loc }}\left((0, T) \times \mathbb{R}^{d}\right)$ means that $f \in L_{p}\left((0, T) \times B_{R}\right)$ for any $R>0$.

In this paper, we use maximal functions and strong maximal functions defined, respectively, by

$$
\mathcal{M} f(t, x)=\sup _{Q_{r}(s, y) \ni(t, x)} f_{Q_{r}(s, y)}|f(r, z)| \chi_{\mathcal{D}} d z d r
$$

and

$$
(\mathcal{S M} f)(t, x)=\sup _{Q_{r_{1}, r_{2}}(s, y) \ni(t, x)} f_{Q_{r_{1}, r_{2}}(s, y)}|f(r, z)| \chi_{\mathcal{D}} d z d r
$$

if $f$ is defined on $\mathcal{D} \subset \mathbb{R}^{d+1}$.

We now present our assumptions for the coefficients. Throughout the paper we assume that there exists $\delta \in(0,1)$ such that

$$
a^{i j}(t, x) \xi_{i} \xi_{j} \geq \delta|\xi|^{2}, \quad\left|a^{i j}\right| \leq \delta^{-1}
$$

for any $(t, x) \in \mathbb{R}^{d+1}$ and $\xi \in \mathbb{R}^{d}$. Without loss of generality, we assume that $a^{i j}=a^{j i}$. $\mathbb{R}^{d}$.

We impose the following regularity assumption on $a^{i j}(t, x)$ with respect to $x \in$

Assumption $2.1\left(\gamma_{0}\right)$. There is a constant $R_{0} \in(0,1]$ such that, for each parabolic cylinder $Q_{r}\left(t_{0}, x_{0}\right)=\left(t_{0}-r^{2 / \alpha}, t_{0}\right) \times B_{r}\left(x_{0}\right)$ with $r \leq R_{0}$ and $\left(t_{0}, x_{0}\right) \in \mathbb{R}^{d+1}$, we have

where

$$
\sup _{i, j} f_{Q_{r}\left(t_{0}, x_{0}\right)}\left|a^{i j}-\bar{a}^{i j}(t)\right| d x d t \leq \gamma_{0},
$$

$$
\bar{a}^{i j}(t)=f_{B_{r}\left(x_{0}\right)} a^{i j}(t, y) d y .
$$


For the lower-order coefficients $b^{i}$ and $c$, we impose the following boundedness condition

$$
\left|b^{i}\right| \leq K_{0}, \quad|c| \leq K_{0},
$$

where $K_{0}$ is a positive constant.

Here is the main theorem of the paper.

Theorem 2.2. Let $\alpha \in(0,1], T \in(0, \infty), p, q \in(1, \infty), K_{1} \in[1, \infty), w=$ $w_{1}(t) w_{2}(x)$, where

$$
w_{1}(t) \in A_{p}(\mathbb{R}, d t), \quad w_{2}(x) \in A_{q}\left(\mathbb{R}^{d}, d x\right), \quad\left[w_{1}\right]_{A_{p}} \leq K_{1}, \quad\left[w_{2}\right]_{A_{q}} \leq K_{1} .
$$

There exists $\gamma_{0}=\gamma_{0}\left(d, \delta, \alpha, p, q, K_{1}\right) \in(0,1)$ such that, under Assumption 2.1] $\left(\gamma_{0}\right)$, the following hold.

For any $u \in \mathbb{H}_{p, q, w, 0}^{\alpha, 2}\left((0, T) \times \mathbb{R}^{d}\right)$ satisfying

$$
-\partial_{t}^{\alpha} u+a^{i j} D_{i j} u+b^{i} D_{i} u+c u=f
$$

in $(0, T) \times \mathbb{R}^{d}$, we have

$$
\left\|\partial_{t}^{\alpha} u\right\|_{p, q, w}+\|u\|_{p, q, w}+\|D u\|_{p, q, w}+\left\|D^{2} u\right\|_{p, q, w} \leq N\|f\|_{p, q, w},
$$

where $\|\cdot\|_{p, q, w}=\|\cdot\|_{L_{p, q, w}\left((0, T) \times \mathbb{R}^{d}\right)}$ and

$$
N=N\left(d, \delta, \alpha, p, q, K_{1}, K_{0}, R_{0}, T\right) .
$$

Moreover, for any $f \in L_{p, q, w}\left((0, T) \times \mathbb{R}^{d}\right)$, there exists a unique solution $u \in$ $\left.\mathbb{H}_{p, q, w, 0}^{\alpha, 2}\left((0, T) \times \mathbb{R}^{d}\right)\right)$ satisfying (2.4) .

\section{Mean oscillation estimates for EQuations With Local time DERIVATIVE}

Throughout the section we assume that $a^{i j}=a^{i j}(t)$, that is, functions of only $t$, satisfying the ellipticity condition (2.3). Let $T \in(-\infty, \infty]$ and $1<p_{0}<\infty$. Let $u \in W_{p_{0}, \text { loc }}^{1,2}\left((-\infty, T) \times \mathbb{R}^{d}\right)$ be a solution to

$$
-\partial_{t} u+a^{i j} D_{i j} u=f
$$

in $(-\infty, T) \times \mathbb{R}^{d}$. For $t_{0} \in(-\infty, T] \cap \mathbb{R}$ and $r>0$, we decompose $u=v+w$ in $\left(-\infty, t_{0}\right) \times B_{r}$, where $w \in W_{p_{0}}^{1,2}\left(\left(-\infty, t_{0}\right) \times B_{r}\right)$ satisfies

$$
-\partial_{t} w+a^{i j}(t) D_{i j} w=f
$$

in $\left(-\infty, t_{0}\right) \times B_{r}$ with the zero boundary condition on $\left(-\infty, t_{0}\right) \times \partial B_{r}$, and $v \in$ $W_{p_{0}}^{1,2}\left(\left(-\infty, t_{0}\right) \times B_{r}\right)$ satisfies

$$
-\partial_{t} v+a^{i j}(t) D_{i j} v=0
$$

in $\left(-\infty, t_{0}\right) \times B_{r}$.

In this section by $Q_{r}\left(t_{0}, x_{0}\right)$ we mean the parabolic cylinder defined in (2.1) with $\alpha=1$. That is,

$$
Q_{r}\left(t_{0}, x_{0}\right)=\left(t_{0}-r^{2}, t_{0}\right) \times B_{r}\left(x_{0}\right) .
$$

By using the usual iteration argument, we obtain the following estimate for $v$ satisfying (3.3). See, for instance, [13, Theorems 5.2.8 and 6.1.1] or [5, Lemma 5.6]. 
Lemma 3.1. Let $p_{0} \in(1, \infty), t_{0} \in \mathbb{R}$, and $v \in W_{p_{0}}^{1,2}\left(\left(-\infty, t_{0}\right) \times B_{r}\right)$ satisfy (3.3) in $\left(-\infty, t_{0}\right) \times B_{r}, r>0$. Then

$$
\left[D^{2} v\right]_{C^{1 / 4,1 / 2}\left(Q_{r / 2}\left(t_{1}, 0\right)\right)} \leq N r^{-1 / 2}\left(\left|D^{2} v\right|^{p_{0}}\right)_{Q_{r}\left(t_{1}, 0\right)}^{1 / p_{0}}
$$

for any $t_{1} \leq t_{0}$, where $N=N\left(d, \delta, p_{0}\right)$.

For $w$ satisfying (3.2), we bound the $L_{p_{0}}$-norm of $D^{2} w$ on $Q_{1 / 2}$ by the sum of the averages of $f$ on cylinders of the form $Q_{r, 1}$, which is in fact bounded by the strong maximal function of $|f|^{p_{0}}$. To obtain such an estimate, we first bound the $L_{p_{0}}$-norm of $w$ on $Q_{1}$ by the sum of such averages.

Lemma 3.2. Let $p_{0} \in(1, \infty), t_{0} \in \mathbb{R}$, and $f \in L_{p_{0}}\left(\left(-\infty, t_{0}\right) \times B_{1}\right)$. Let $w \in$ $W_{p_{0}}^{1,2}\left(\left(-\infty, t_{0}\right) \times B_{1}\right)$ be a solution to (3.2) in $\left(-\infty, t_{0}\right) \times B_{1}$ with the zero boundary condition on $\left(-\infty, t_{0}\right) \times \partial B_{1}$. Then we have

$$
\|w\|_{L_{p_{0}}\left(Q_{1}\left(t_{0}, 0\right)\right)} \leq N \sum_{k=0}^{\infty} c_{k}\left(|f|^{p_{0}}\right)_{\left(t_{0}-2^{k+2}+2, t_{0}\right) \times B_{1}}^{1 / p_{0}},
$$

where $N=N\left(d, \delta, p_{0}\right)$ is independent of $t_{0}$ and $\left\{c_{k}\right\}$ is a sequence satisfying

$$
\sum_{k=0}^{\infty} c_{k} \leq N=N\left(d, \delta, p_{0}\right)
$$

Proof. To derive (3.4), we prove that there exists $\varepsilon=\varepsilon\left(d, \delta, p_{0}\right)>0$ such that

$$
e^{\varepsilon\left(t_{0}-1\right)}\|w\|_{L_{p_{0}}\left(Q_{1}\left(t_{0}, 0\right)\right)} \leq N\left\|e^{\varepsilon t} f(t, x)\right\|_{L_{p_{0}}\left(\left(-\infty, t_{0}\right) \times B_{1}\right)},
$$

where $N=N\left(d, \delta, p_{0}\right)$. Note that

$$
\begin{aligned}
& \left\|e^{\varepsilon t} f(t, x)\right\|_{L_{p_{0}}\left(\left(-\infty, t_{0}\right) \times B_{1}\right)}=\left(\sum_{k=0}^{\infty} \int_{t_{0}-2^{k+2}+2}^{t_{0}-2^{k+1}+2} e^{\varepsilon p_{0} t} \int_{B_{1}}|f(t, x)|^{p_{0}} d x d t\right)^{1 / p_{0}} \\
& \leq\left(\sum_{k=0}^{\infty} \int_{t_{0}-2^{k+2}+2}^{t_{0}-2^{k+1}+2} e^{\varepsilon p_{0}\left(t_{0}-2^{k+1}+2\right)} \int_{B_{1}}|f(t, x)|^{p_{0}} d x d t\right)^{1 / p_{0}} \\
& \leq N(d) e^{\varepsilon t_{0}}\left(\sum_{k=0}^{\infty} e^{\varepsilon p_{0}\left(2-2^{k+1}\right)}\left(2^{k+2}-2\right)\left(|f|^{p_{0}}\right)_{\left(t_{0}-2^{k+2}+2, t_{0}\right) \times B_{1}}\right)^{1 / p_{0}} \\
& \leq N e^{\varepsilon t_{0}} \sum_{k=0}^{\infty} e^{\varepsilon\left(2-2^{k+1}\right)}\left(2^{k+2}-2\right)^{1 / p_{0}}\left(|f|^{p_{0}}\right)_{\left(t_{0}-2^{k+2}+2, t_{0}\right) \times B_{1}}^{1 / p_{0}}
\end{aligned}
$$

Thus, by setting

$$
c_{k}=e^{\varepsilon\left(2-2^{k+1}\right)}\left(2^{k+2}-2\right)^{1 / p_{0}},
$$

which satisfies (3.5), from (3.6) we arrive at (3.4).

For the proof of (3.6), we show that there exists $\varepsilon_{0}=\varepsilon_{0}\left(d, \delta, p_{0}\right)>0$ such that, for $\varepsilon \in\left[0, \varepsilon_{0}\right], U \in W_{p_{0}}^{1,2}\left(\left(-\infty, \tau_{0}\right) \times B_{1}\right)$, and $F \in L_{p_{0}}\left(\left(-\infty, \tau_{0}\right) \times B_{1}\right)$ satisfying

$$
-\partial_{t} U+a^{i j}(t) D_{i j} U+\varepsilon U=F
$$

in $\left(-\infty, \tau_{0}\right) \times B_{1}$ and $U=0$ on $\left(-\infty, \tau_{0}\right) \times \partial B_{1}$, where $\tau_{0} \in(-\infty, \infty]$, we have

$$
\|U\|_{L_{p_{0}}\left(\left(-\infty, \tau_{0}\right) \times B_{1}\right)} \leq N\|F\|_{L_{p_{0}}\left(\left(-\infty, \tau_{0}\right) \times B_{1}\right)},
$$


where $N=N\left(d, \delta, p_{0}\right)$ is independent of $\tau_{0}$. If this holds, one can obtain (3.6) by taking $\varepsilon \in\left[0, \varepsilon_{0}\right], \tau_{0}=t_{0}$,

$$
U=w e^{\varepsilon t} \in W_{p_{0}}^{1,2}\left(\left(-\infty, t_{0}\right) \times B_{1}\right), \quad F=e^{\varepsilon t} f \in L_{p_{0}}\left(\left(-\infty, t_{0}\right) \times B_{1}\right) .
$$

To show (3.8), we consider two cases.

Case 1: $p_{0} \in[2, \infty)$. We multiply both sides of (3.7) by $-p_{0}|U|^{p_{0}-2} U$ and integrate in $\left(-\infty, \tau_{0}\right) \times B_{1}$. By noting that the integral involving $\partial_{t} U$ is nonnegative, we have

$$
\begin{gathered}
\int_{-\infty}^{\tau_{0}} \int_{B_{1}} a_{i j} p_{0}\left(p_{0}-1\right)|U|^{p_{0}-2} D_{j} U D_{i} U d x d t-\varepsilon p_{0} \int_{-\infty}^{\tau_{0}} \int_{B_{1}}|U|^{p_{0}} d x d t \\
\leq \int_{-\infty}^{\tau_{0}} \int_{B_{1}} p_{0}|F||U|^{p_{0}-1} d x d t .
\end{gathered}
$$

By using the zero boundary condition and the Poincare inequality to $G(U)=$ $|U|^{p_{0} / 2}$ with respect to the spatial variables, as well as using the ellipticity condition, from the above inequality we get

$$
\begin{aligned}
& \int_{-\infty}^{\tau_{0}} \int_{B_{1}}|U|^{p_{0}} d x d t=\int_{-\infty}^{\tau_{0}} \int_{B_{1}}|G(U)|^{2} d x d t \\
& \leq N \int_{-\infty}^{\tau_{0}} \int_{B_{1}}|D(G(U))|^{2} d x d t \leq N \int_{-\infty}^{\tau_{0}} \int_{B_{1}} \frac{p_{0}^{2}}{4}|D U|^{2}|U|^{p_{0}-2} d x d t \\
& \leq N \int_{-\infty}^{\tau_{0}} \int_{B_{1}} p_{0}\left(p_{0}-1\right)|D U|^{2}|U|^{p_{0}-2} d x d t \\
& \leq N \int_{-\infty}^{\tau_{0}} \int_{B_{1}} e^{\varepsilon t}|F||U|^{p_{0}-1} d x d t+N \varepsilon \int_{-\infty}^{\tau_{0}} \int_{B_{1}}|U|^{p_{0}} d x d t,
\end{aligned}
$$

where $N=N\left(d, \delta, p_{0}\right)$. Upon choosing a sufficiently small $\varepsilon_{0}=\varepsilon_{0}\left(d, \delta, p_{0}\right)>0$, from the above inequalities and Young's inequality, we arrive at (3.8) whenever $\varepsilon \in\left[0, \varepsilon_{0}\right]$.

Case 2: $p_{0} \in(1,2)$. We use a duality argument. Take $\varepsilon_{0}$ from Case 1 and set $q_{0}=p_{0} /\left(p_{0}-1\right) \in(2, \infty)$. For $\varepsilon \in\left[0, \varepsilon_{0}\right]$ and $g \in C_{0}^{\infty}\left(\left(-\infty, \tau_{0}\right) \times B_{1}\right)$, let $U_{1}$ be the unique solution in $W_{q_{0}}^{1,2}\left(\left(-\tau_{0}, \infty\right) \times B_{1}\right)$ of the equation

$$
-\partial_{t} U_{1}+a^{i j}(-t) D_{i j} U_{1}+\varepsilon U_{1}=g(-t, x)
$$

in $\left(-\tau_{0}, \infty\right) \times B_{1}$ with the zero boundary condition on $\partial_{p}\left(\left(-\tau_{0}, \infty\right) \times B_{1}\right)$. Indeed, to obtain the existence of such a solution $U_{1}$, we solve

$$
-\partial_{t} U_{1}+a^{i j}(-t) D_{i j} U_{1}+\varepsilon U_{1}=g(-t, x) \chi_{\left(-t_{0}, \infty\right)}
$$

in $\mathbb{R} \times B_{1}$ with the zero boundary condition on $\mathbb{R} \times \partial B_{1}$. The solvability of this equation is guaranteed by the usual $L_{p}$-theory for parabolic equations with coefficients measurable in time (see, for instance, 4, 9]) and the estimate

$$
\left\|U_{1}\right\|_{L_{q_{0}}\left(\left(-\tau_{0}, \infty\right) \times B_{1}\right)} \leq N\|g(-\cdot, \cdot)\|_{L_{q_{0}}\left(\left(-\tau_{0}, \infty\right) \times B_{1}\right)},
$$

proved in Case 1 when the time interval is $\mathbb{R}$. One can check the zero initial condition of $U_{1}$ at $t=-\tau_{0}$ by noting that the solution $U_{1}$ to the equation (3.9) is zero for $t \leq-\tau_{0}$. Then by using integration by parts, we have

$$
\int_{-\infty}^{t_{0}} \int_{B_{1}} U g d x d t=\int_{-\infty}^{t_{0}} \int_{B_{1}} F(t, x) U_{1}(-t, x) d x d t
$$

This together with (3.10) immediately gives (3.8). The lemma is proved. 
Lemma 3.3. Let $p_{0} \in(1, \infty), t_{0} \in \mathbb{R}$, and $f \in L_{p_{0}}\left(\left(-\infty, t_{0}\right) \times B_{1}\right)$. Let $w \in$ $W_{p_{0}}^{1,2}\left(\left(-\infty, t_{0}\right) \times B_{1}\right)$ be a solution to (3.2) in $\left(-\infty, t_{0}\right) \times B_{1}$ with the zero boundary condition on $\left(-\infty, t_{0}\right) \times \partial B_{1}$. Then we have

$$
\left(\left|D^{2} w\right|^{p_{0}}\right)_{Q_{1 / 2}\left(t_{1}, 0\right)}^{1 / p_{0}} \leq N \sum_{k=0}^{\infty} c_{k}\left(|f|^{p_{0}}\right)_{\left(t_{0}-2^{k+2}+2, t_{0}\right) \times B_{1}}^{1 / p_{0}},
$$

where $N=N\left(d, \delta, p_{0}\right)$ and $\left\{c_{k}\right\}$ satisfies (3.5).

Proof. Since the assumptions are satisfied if $t_{0}$ is replaced with $t_{1}$, we prove only the case $t_{1}=t_{0}$. By using the standard local $L_{p_{0}}$-estimate for parabolic equations with coefficients measurable in time (see, for instance, [13, Theorem 6.4.2]), we have

$$
\left(\left|D^{2} w\right|^{p_{0}}\right)_{Q_{1 / 2}\left(t_{0}, 0\right)}^{1 / p_{0}} \leq N\left(|f|^{p_{0}}\right)_{Q_{1}\left(t_{0}, 0\right)}^{1 / p_{0}}+N\left(|w|^{p_{0}}\right)_{Q_{1}\left(t_{0}, 0\right)}^{1 / p_{0}}
$$

where $N=N\left(d, \delta, p_{0}\right)$. This combined with Lemma 3.2 proves (3.11). The lemma is proved.

Proposition 3.4. Let $p_{0} \in(1, \infty), T \in(-\infty, \infty]$, and $u \in W_{p_{0}, \text { loc }}^{1,2}\left((-\infty, T) \times \mathbb{R}^{d}\right)$ satisfy (3.1) in $(-\infty, T) \times \mathbb{R}^{d}$. Then, for any $\left(t_{0}, x_{0}\right) \in(-\infty, T] \times \mathbb{R}^{d}$ with $t_{0} \in \mathbb{R}$, $r \in(0, \infty)$, and $\kappa \in(0,1 / 4)$, we have

$$
\begin{aligned}
& \left(\left|D^{2} u-\left(D^{2} u\right)_{Q_{\kappa r}\left(t_{0}, x_{0}\right)}\right|\right)_{Q_{\kappa r}\left(t_{0}, x_{0}\right)} \\
& \leq N \kappa^{1 / 2}\left(\left|D^{2} u\right|^{p_{0}}\right)_{Q_{r}\left(t_{0}, x_{0}\right)}^{1 / p_{0}}+N \kappa^{-(d+2) / p_{0}} \sum_{k=0}^{\infty} c_{k}\left(|f|^{p_{0}}\right)_{\left(t_{0}-\left(2^{k+2}-2\right) r^{2}, t_{0}\right) \times B_{r}\left(x_{0}\right)}^{1 / p_{0}},
\end{aligned}
$$

where $N=N\left(d, \delta, p_{0}\right)$ and $\left\{c_{k}\right\}$ satisfies (3.5).

Proof. Thanks to translation with respect to the spatial variables and dilation, we assume that $x_{0}=0$ and $r=1$. Since $u \in W_{p_{0}, \text { loc }}^{1,2}\left((-\infty, T) \times \mathbb{R}^{d}\right)$, we have $f \in L_{p_{0}}\left((-\infty, T) \times B_{1}\right)$. Thus one can find $w \in W_{p_{0}}^{1,2}\left(\left(-\infty, t_{0}\right) \times B_{1}\right)$ satisfying (3.2) in $\left(-\infty, t_{0}\right) \times B_{1}$ with the zero boundary condition on $\left(-\infty, t_{0}\right) \times \partial B_{1}$. We note that the solvability follows from (3.8), the interior and boundary $W_{p}^{1,2}$-estimates (see, for instance, [4), and the method of continuity. Set $v=u-w$. Then $v$ belongs to $W_{p_{0}}^{1,2}\left(\left(-\infty, t_{0}\right) \times B_{1}\right)$ and satisfies (3.3) in $\left(-\infty, t_{0}\right) \times B_{1}$. Since $\kappa<1 / 4$, by Lemma 3.1 with $r=1 / 2$ and the fact that $u=v+w$, we observe that

$$
\begin{aligned}
& \left(\left|D^{2} v-\left(D^{2} v\right)_{Q_{\kappa}\left(t_{0}, 0\right)}\right|\right)_{Q_{\kappa}\left(t_{0}, 0\right)} \leq 3 \kappa^{1 / 2}\left[D^{2} v\right]_{C^{1 / 4,1 / 2}\left(Q_{1 / 4}\left(t_{0}, 0\right)\right)} \\
& \leq N \kappa^{\frac{1}{2}}\left(\left|D^{2} v\right|^{p_{0}}\right)_{Q_{\frac{1}{2}}^{\frac{1}{p_{0}}}\left(t_{0}, 0\right)} \leq N \kappa^{\frac{1}{2}}\left(\left|D^{2} u\right|^{p_{0}}\right)_{Q_{\frac{1}{2}}^{\frac{1}{p_{0}}}\left(t_{0}, 0\right)}+N \kappa^{\frac{1}{2}}\left(\left|D^{2} w\right|^{p_{0}}\right)_{Q_{\frac{1}{2}}^{\frac{1}{p_{0}}}}^{\frac{1}{\left.t_{0}, 0\right)}} .
\end{aligned}
$$

This combined with Lemma 3.3 and the triangle inequality shows that

$$
\begin{aligned}
& \left(\left|D^{2} u-\left(D^{2} u\right)_{Q_{\kappa}\left(t_{0}, 0\right)}\right|\right)_{Q_{\kappa}\left(t_{0}, 0\right)} \\
& \leq\left(\left|D^{2} v-\left(D^{2} v\right)_{Q_{\kappa}\left(t_{0}, 0\right)}\right|\right)_{Q_{\kappa}\left(t_{0}, 0\right)}+N\left(\left|D^{2} w\right|\right)_{Q_{\kappa}\left(t_{0}, 0\right)} \\
& \leq N \kappa^{\frac{1}{2}}\left(\left|D^{2} u\right|^{p_{0}}\right)_{Q_{\frac{1}{2}}^{\frac{1}{p_{0}}}\left(t_{0}, 0\right)}+N \kappa^{-\frac{d+2}{p_{0}}}\left(\left|D^{2} w\right|^{p_{0}}\right)_{Q_{\frac{1}{2}}^{p_{0}}}^{\frac{1}{\left.p_{0}, 0\right)}} \\
& \leq N \kappa^{\frac{1}{2}}\left(\left|D^{2} u\right|^{p_{0}}\right)_{Q_{1}}^{\frac{1}{p_{0}}}\left(t_{0}, 0\right) \\
& +N \kappa^{-\frac{d+2}{p_{0}}} \sum_{k=0}^{\infty} c_{k}\left(|f|^{p_{0}}\right)_{\left(t_{0}-2^{k+2}+2, t_{0}\right) \times B_{1}}^{1 / p_{0}},
\end{aligned}
$$

where $N=N\left(d, \delta, p_{0}\right)$. The proposition is proved. 


\section{Mean oscillation estimates for EQUATions With NON-LOCAL Time DERIVATIVE}

Throughout the section we assume that $\alpha \in(0,1)$ and $a^{i j}=a^{i j}(t)$, that is, functions of only $t$, satisfying the ellipticity condition (2.3). Let $p_{0} \in(1, \infty)$ and $T \in(0, \infty)$. Let $u \in \mathbb{H}_{p_{0}, 0, \text { loc }}^{\alpha, 2}\left((0, T) \times \mathbb{R}^{d}\right)$ be a solution to

$$
-\partial_{t}^{\alpha} u+a^{i j}(t) D_{i j} u=f(t, x)
$$

in $(0, T) \times \mathbb{R}^{d}$. Note that the zero initial condition $u(0, \cdot)=0$ is implicitly imposed because $u \in \mathbb{H}_{p_{0}, 0, \text { loc }}^{\alpha, 2}\left((0, T) \times \mathbb{R}^{d}\right)$.

For convenience, we extend $u$ and $f$ to be zero when $t \leq 0$. For $t_{0} \in(0, T]$ and $r>0$, we decompose $u=v+w$ in $\left(0, t_{0}\right) \times B_{r}$, where $w$ is a weak solution to

$$
-\partial_{t}^{\alpha} w+a^{i j}(t) D_{i j} w=f(t, x)
$$

in $\left(0, t_{0}\right) \times B_{r}$ with the zero boundary condition on $\partial_{p}\left(\left(0, t_{0}\right) \times B_{r}\right)$, and $v$ satisfies

$$
-\partial_{t}^{\alpha} v+a^{i j}(t) D_{i j} v=0
$$

in $\left(0, t_{0}\right) \times B_{r}$. Throughout the section, recall that, for $\alpha \in(0,1)$,

$$
Q_{r}\left(t_{0}, x_{0}\right)=\left(t_{0}-r^{2 / \alpha}, t_{0}\right) \times B_{r}\left(x_{0}\right) .
$$

\subsection{Estimates of $v$.}

Lemma 4.1. Let $p_{0} \in(1, \infty), t_{0} \in(0, \infty)$, and $v \in \mathbb{H}_{p_{0}, 0}^{\alpha, 2}\left(\left(0, t_{0}\right) \times B_{r}\right)$ satisfy (4.3) in $\left(0, t_{0}\right) \times B_{r}, r>0$. Then there exists

$$
p_{1}=p_{1}\left(d, \alpha, p_{0}\right) \in\left(p_{0}, \infty\right]
$$

satisfying

$$
p_{1}>p_{0}+\min \left\{\frac{2 \alpha}{\alpha d+2-2 \alpha}, \alpha, \frac{2}{d}\right\}
$$

such that

$$
\left(\left|D^{2} v\right|^{p_{1}}\right)_{Q_{r / 2}\left(t_{1}, 0\right)}^{1 / p_{1}} \leq N \sum_{j=1}^{\infty} j^{-(1+\alpha)}\left(\left|D^{2} v\right|^{p_{0}}\right)_{Q_{r}\left(t_{1}-(j-1) r^{2 / \alpha}, 0\right)}^{1 / p_{0}}
$$

for any $t_{1} \leq t_{0}$, where $N=N\left(d, \delta, \alpha, p_{0}\right)$ and

$$
\left(\left|D^{2} v\right|^{p_{1}}\right)_{Q_{r / 2}\left(t_{1}, 0\right)}^{1 / p_{1}}=\left\|D^{2} v\right\|_{L_{\infty}\left(Q_{r / 2}\left(t_{1}, 0\right)\right)} \quad \text { if } \quad p_{1}=\infty .
$$

If $p_{0}>d / 2+1 / \alpha$, then

$$
\left[D^{2} v\right]_{C^{\sigma \alpha / 2, \sigma}\left(Q_{r / 2}\left(t_{1}, 0\right)\right)} \leq N r^{-\sigma} \sum_{j=1}^{\infty} j^{-(1+\alpha)}\left(\left|D^{2} v\right|^{p_{0}}\right)_{Q_{r}\left(t_{1}-(j-1) r^{2 / \alpha}, 0\right)}^{1 / p_{0}}
$$

for any $t_{1} \leq t_{0}$, where $\sigma=\sigma\left(d, \alpha, p_{0}\right) \in(0,1)$. Moreover, if $p_{1}<\infty$, then $v \in$ $\mathbb{H}_{p_{1}, 0}^{\alpha, 2}\left(\left(0, t_{0}\right) \times B_{r / 2}\right)$.

Proof. Thanks to scaling, we only need to prove the assertions for $r=1$. Note that $v$ can be extended as zero for $t \leq 0$. Thus by Lemma 3.5 in [6] $v$ belongs to $\mathbb{H}_{p_{0}, 0}^{\alpha, 2}\left(\left(S, t_{0}\right) \times B_{1}\right)$ for any $S \leq 0$. We also see that $v$ satisfies (4.3) in $\left(S, t_{0}\right) \times B_{1}$.

Find an infinitely differentiable function $\eta$ defined on $\mathbb{R}$ such that

$$
\eta= \begin{cases}1 & \text { if } \quad t \in\left(t_{1}-(1 / 2)^{2 / \alpha}, t_{1}\right) \\ 0 & \text { if } \quad t \in \mathbb{R} \backslash\left(t_{1}-1, t_{1}+1\right)\end{cases}
$$


and

$$
\left|\frac{\eta(t)-\eta(s)}{t-s}\right| \leq N(\alpha) .
$$

Then by Lemmas 3.6 and 4.4 (and the proof of the latter lemma) in [6],

$$
\eta v, D(\eta v), D^{2}(\eta v) \in \mathbb{H}_{p_{0}, 0}^{\alpha, 2}\left(\left(t_{1}-1, t_{1}\right) \times B_{3 / 4}\right)
$$

and $D^{2}(\eta v)$ satisfies

$$
-\partial_{t}^{\alpha}\left(D^{2}(\eta v)\right)+a^{i j} D_{i j} D^{2}(\eta v)=\mathcal{G}
$$

in $\left(t_{1}-1, t_{1}\right) \times B_{3 / 4}$, where $\partial_{t}^{\alpha}=\partial_{t} I_{t_{1}-1}^{1-\alpha}$ and

$$
\mathcal{G}(t, x)=\frac{\alpha}{\Gamma(1-\alpha)} \int_{-\infty}^{t}(t-s)^{-\alpha-1}(\eta(t)-\eta(s)) D^{2} v(s, x) d s .
$$

If $p \leq 1 / \alpha$, take $p_{1}$ satisfying

$$
\begin{gathered}
p_{1} \in\left(p_{0}, \frac{1 / \alpha+d / 2}{1 /\left(\alpha p_{0}\right)+d /\left(2 p_{0}\right)-1}\right) \quad \text { if } \quad p_{0} \leq d / 2, \\
p_{1} \in\left(p_{0}, p_{0}\left(\alpha p_{0}+1\right)\right) \quad \text { if } \quad p_{0}>d / 2 .
\end{gathered}
$$

If $p_{0}>1 / \alpha$, take $p_{1}$ satisfying

$$
\begin{gathered}
p_{1} \in\left(p_{0}, p_{0}+2 p_{0}^{2} / d\right) \quad \text { if } \quad p_{0} \leq d / 2, \\
p_{1} \in\left(p_{0}, 2 p_{0}\right) \quad \text { if } \quad p_{0}>d / 2, \quad p_{0} \leq d / 2+1 / \alpha, \\
p_{1}=\infty \quad \text { if } \quad p_{0}>d / 2+1 / \alpha .
\end{gathered}
$$

Note that $p_{1}$ satisfies (4.4) and the increment $\min \{2 \alpha /(\alpha d+2-2 \alpha), \alpha, 2 / d\}$ is independent of $p_{0}$. By (4.7) and the Sobolev type embeddings obtained in [6] we have

$$
\eta v, D(\eta v), D^{2}(\eta v) \in L_{p_{1}}\left(\left(t_{1}-1, t_{1}\right) \times B_{3 / 4}\right) .
$$

By the Sobolev embeddings again and Lemma 4.2 in [6] for local $L_{p}$-estimates, we have

$$
\begin{aligned}
& \left.\left\|D^{2} v\right\|_{L_{p_{1}}\left(Q_{1 / 2}\left(t_{1}, 0\right)\right)} \leq\left\|D^{2}(\eta v)\right\|_{L_{p_{1}}\left(\left(t_{1}-1, t_{1}\right) \times B_{1 / 2}\right.}\right) \\
& \left.\leq N\left\|\left|D^{2}(\eta v)\right|+\left|D^{4}(\eta v)\right|+\left|D_{t}^{\alpha} D^{2}(\eta v)\right|\right\|_{L_{p_{0}}\left(\left(t_{1}-1, t_{1}\right) \times B_{3 / 4}\right.}\right) \\
& \leq N||\left|D^{2}(\eta v)\right|+|\mathcal{G}|\left\|_{L_{p_{0}}\left(\left(t_{1}-1, t_{1}\right) \times B_{1}\right)} \leq N\right\|\left|D^{2} v\right|+|\mathcal{G}| \|_{L_{p_{0}}\left(\left(t_{1}-1, t_{1}\right) \times B_{1}\right)},
\end{aligned}
$$

where $N=N\left(d, \delta, \alpha, p_{0}, p_{1}\right)$ and we used (4.8). We write

$$
\begin{aligned}
& \frac{\Gamma(1-\alpha)}{\alpha} \mathcal{G}(t, x)=\int_{t-1}^{t}(t-s)^{-\alpha-1}(\eta(s)-\eta(t)) D^{2} v(s, x) d s \\
& \quad+\int_{-\infty}^{t-1}(t-s)^{-\alpha-1}(\eta(s)-\eta(t)) D^{2} v(s, x) d s:=I_{1}(t, x)+I_{2}(t, x),
\end{aligned}
$$

where

$$
\left|I_{1}(t, x)\right| \leq N \int_{t-1}^{t}|t-s|^{-\alpha}\left|D^{2} v(s, x)\right| d s=N \int_{0}^{1}|s|^{-\alpha}\left|D^{2} v(t-s, x)\right| d s,
$$

which implies

$$
\left\|I_{1}\right\|_{L_{p_{0}}\left(\left(t_{1}-1, t_{1}\right) \times B_{1}\right)} \leq N\left\|D^{2} v\right\|_{L_{p_{0}}\left(\left(t_{1}-2, t_{1}\right) \times B_{1}\right)} .
$$


To estimate $I_{2}$, we see that $\eta(s)=0$ for any $s \in(-\infty, t-1)$ with $t \in\left(t_{1}-1, t_{1}\right)$. Thus we have

$$
I_{2}(t, x)=-\eta(t) \int_{-\infty}^{t-1}(t-s)^{-\alpha-1} D^{2} v(s, x) d s .
$$

Then,

$$
\begin{aligned}
\left|I_{2}(t, x)\right| & \leq \int_{-\infty}^{t-1}|t-s|^{-\alpha-1}\left|D^{2} v(s, x)\right| d s \\
& =\sum_{j=1}^{\infty} \int_{t-j-1}^{t-j}|t-s|^{-\alpha-1}\left|D^{2} v(s, x)\right| d s \leq \sum_{j=1}^{\infty} \int_{t-j-1}^{t-j} j^{-(\alpha+1)}\left|D^{2} v(s, x)\right| d s .
\end{aligned}
$$

From this we have

$$
\left\|I_{2}\right\|_{L_{p_{0}}\left(\left(t_{1}-1, t_{1}\right) \times B_{1}\right)} \leq \sum_{j=1}^{\infty} j^{-(\alpha+1)}\left\|\int_{t-j-1}^{t-j}\left|D^{2} v(s, x)\right| d s\right\|_{L_{p_{0}}\left(\left(t_{1}-1, t_{1}\right) \times B_{1}\right)} .
$$

Since $t_{1}-1<t<t_{1}$,

$$
\int_{t-j-1}^{t-j}\left|D^{2} v(s, x)\right| d s \leq \int_{t_{1}-j-2}^{t_{1}-j}\left|D^{2} v(s, x)\right| d s .
$$

Hence, by Hölder's inequality,

$$
\left\|I_{2}\right\|_{L_{p_{0}}\left(Q_{1}\left(t_{1}, 0\right)\right)} \leq N \sum_{j=1}^{\infty} j^{-(\alpha+1)}\left\|D^{2} v\right\|_{L_{p_{0}}\left(\left(t_{1}-j-2, t_{1}-j\right) \times B_{1}\right)} .
$$

Combining the above inequality, (4.10), and (4.11), we reach (4.5) with $r=1$.

For the proof of (4.6), if $p_{0}>d / 2+1 / \alpha$, we find $\tilde{p}_{0}$ such that $\tilde{p}_{0} \leq p_{0}$ and $\tilde{p}_{0} \in(d / 2+1 / \alpha, d+2 / \alpha)$. By (4.7) and the Sobolev embeddings in [6], we have

$$
D^{2}(\eta v) \in C^{\sigma \alpha / 2, \sigma}\left(\left(t_{1}-1, t_{1}\right) \times B_{3 / 4}\right),
$$

where $\sigma=2-(d+2 / \alpha) / \tilde{p}_{0} \in(0,1)$. We then repeat the above steps from the inequalities in (4.10) with $\left[D^{2} v\right]_{C^{\sigma \alpha / 2, \sigma}\left(Q_{1 / 2}\left(t_{0}, 0\right)\right)}$ in place of $\left\|D^{2} v\right\|_{L_{p_{1}}\left(Q_{1 / 2}\left(t_{0}, 0\right)\right)}$.

We now show that $v \in \mathbb{H}_{p_{1}, 0}^{\alpha, 2}\left(\left(0, t_{0}\right) \times B_{1 / 2}\right)$ when $p_{1}<\infty$. From (4.9) and the equation (4.3) it follows that

$$
v, D v, D^{2} v, \partial_{t}^{\alpha} v \in L_{p_{1}}\left(\left(0, t_{0}\right) \times B_{3 / 4}\right) .
$$

Note that as mentioned in [6, Remark 3.4] this is not enough even to claim that $v$ belongs to $\mathbb{H}_{p_{1}}^{\alpha, 2}\left(\left(0, t_{0}\right) \times B_{1 / 2}\right)$, which is a superset of $\mathbb{H}_{p_{1}, 0}^{\alpha, 2}\left(\left(0, t_{0}\right) \times B_{1 / 2}\right)$. We take the mollification $v^{(\varepsilon)}$ of $v$ given in the proof of Proposition 3.2 in [6]. That is, we use

$$
v^{(\varepsilon)}(t, x)=\int_{0}^{t_{0}} \int_{B_{1}} \eta_{\varepsilon}(t-s, x-y) v(s, y) I_{0<s<t_{0}} d y d s,
$$

where $\eta_{\varepsilon}(t, x)=\varepsilon^{-d-2 / \alpha} \eta\left(t / \varepsilon^{2 / \alpha}, x / \varepsilon\right), \eta(t, x)$ is an infinitely differentiable function defined in $\mathbb{R}^{d+1}$ with compact support in $(0,1) \times B_{1}$ and $\int_{\mathbb{R}^{d+1}} \eta d x d t=1$. By using the fact that $\eta(t, x)=0$ for $t \leq 0$ and $v \in \mathbb{H}_{p_{0}, 0}^{\alpha, 2}\left(\left(0, t_{0}\right) \times B_{1}\right)$, one can check that $v^{(\varepsilon)}(0, x)=0$ and, for $(t, x) \in\left(0, t_{0}\right) \times B_{1 / 2}$,

$$
\partial_{t}^{\alpha} v^{(\varepsilon)}(t, x)=\int_{0}^{t_{0}} \int_{B_{1}} \eta_{\varepsilon}(t-s, x-y) \partial_{t}^{\alpha} v(s, y) d y d s,
$$




$$
D_{x}^{m} v^{(\varepsilon)}(t, x)=\int_{0}^{t_{0}} \int_{B_{1}} \eta_{\varepsilon}(t-s, x-y) D_{x}^{m} v(s, y) d y d s, \quad m=0,1,2 .
$$

Then

$$
\left\|v^{(\varepsilon)}-v\right\|_{\mathbb{H}_{p 1}^{\alpha, 2}\left(\left(0, t_{0}\right) \times B_{1 / 2}\right)} \rightarrow 0
$$

as $\varepsilon \rightarrow 0$. This implies that $v \in \mathbb{H}_{p_{1}, 0}^{\alpha, 2}\left(\left(0, t_{0}\right) \times B_{1 / 2}\right)$. The lemma is proved.

We need the following simple inequality.

Lemma 4.2. For any $\alpha>0$ and $k=2,3, \ldots$, we have

$$
\sum_{j=1}^{k-1} j^{-(1+\alpha)}(k-j)^{-(1+\alpha)} \leq N(\alpha) k^{-(1+\alpha)} .
$$

Proof. By using Hölder's inequality on $(1 / j+1 /(k-j))^{1+\alpha}$, we have

$$
\begin{aligned}
& \sum_{j=1}^{k-1} k^{(1+\alpha)} j^{-(1+\alpha)}(k-j)^{-(1+\alpha)}=\sum_{j=1}^{k-1}(1 / j+1 /(k-j))^{1+\alpha} \\
& \leq 2^{\alpha} \sum_{j=1}^{k}\left(1 / j^{1+\alpha}+1 /(k-j)^{1+\alpha}\right) \leq N(\alpha) .
\end{aligned}
$$

The lemma is proved.

Proposition 4.3. Let $1<p_{0}<p<\infty, t_{0} \in(0, \infty)$, and $v \in \mathbb{H}_{p_{0}, 0}^{\alpha, 2}\left(\left(0, t_{0}\right) \times B_{r}\right)$ satisfy (4.3) in $\left(0, t_{0}\right) \times B_{r}, r>0$. Then we have

$$
\left(\left|D^{2} v\right|^{p}\right)_{Q_{r / 2}\left(t_{1}, 0\right)}^{1 / p} \leq N \sum_{j=1}^{\infty} j^{-(1+\alpha)}\left(\left|D^{2} v\right|^{p_{0}}\right)_{Q_{r}\left(t_{1}-(j-1) r^{2 / \alpha}, 0\right)}^{1 / p_{0}}
$$

for any $t_{1} \leq t_{0}$, where $N=N\left(d, \delta, \alpha, p, p_{1}\right)$. Furthermore,

$$
\left[D^{2} v\right]_{C^{\sigma \alpha / 2, \sigma}\left(Q_{r / 2}\left(t_{1}, 0\right)\right)} \leq N r^{-\sigma} \sum_{j=1}^{\infty} j^{-(1+\alpha)}\left(\left|D^{2} v\right|^{p_{0}}\right)_{Q_{r}\left(t_{1}-(j-1) r^{2 / \alpha}, 0\right)}^{1 / p_{0}}
$$

for any $t_{1} \leq t_{0}$, where $\sigma=\sigma\left(d, \alpha, p_{0}\right) \in(0,1)$.

Proof. Due to scaling, we consider only the case $r=1$. By Lemma 4.1. one can find $p_{1}$ satisfying (4.4) such that

$$
\left(\left|D^{2} v\right|^{p_{1}}\right)_{Q_{1 / 2}(t, 0)}^{1 / p_{1}} \leq N \sum_{j=1}^{\infty} j^{-(1+\alpha)}\left(\left|D^{2} v\right|^{p_{0}}\right)_{Q_{1}(t-j+1,0)}^{1 / p_{0}}
$$

for any $t \leq t_{0}$. If $p_{1} \geq p$, we reach (4.12). Otherwise, by the same lemma, $v \in \mathbb{H}_{p_{1}, 0}^{\alpha, 2}\left(\left(0, t_{0}\right) \times B_{1 / 2}\right)$ and there exists $p_{2}$ satisfying

$$
p_{2}>p_{1}+\min \left\{\frac{2 \alpha}{\alpha d+2-2 \alpha}, \alpha, \frac{2}{d}\right\}
$$

and

$$
\left(\left|D^{2} v\right|^{p_{2}}\right)_{Q_{1 / 4}\left(t_{1}, 0\right)}^{1 / p_{2}} \leq N \sum_{j=1}^{\infty} j^{-(1+\alpha)}\left(\left|D^{2} v\right|^{p_{1}}\right)_{Q_{1 / 2}\left(t_{1}-(j-1) 2^{-2 / \alpha}, 0\right)}^{1 / p_{1}} .
$$


Combining (4.14) and (4.15), we get

$$
\begin{aligned}
\left(\left|D^{2} v\right|^{p_{2}}\right)_{Q_{1 / 4}\left(t_{1}, 0\right)}^{1 / p_{2}} & \leq N \sum_{j=1}^{\infty} j^{-(1+\alpha)}\left(\left|D^{2} v\right|^{p_{1}}\right)_{Q_{1 / 2}\left(t_{1}-(j-1) 2^{-2 / \alpha}, 0\right)}^{1 / p_{1}} \\
& \leq N \sum_{j=1}^{\infty} j^{-(1+\alpha)} \sum_{k=1}^{\infty} k^{-(1+\alpha)}\left(\left|D^{2} v\right|^{p_{0}}\right)_{Q_{1}\left(t_{1}-(j-1) 2^{-2 / \alpha}-k+1,0\right)}^{1 / p_{0}}
\end{aligned}
$$

Note that

$$
\begin{aligned}
& \sum_{j=1}^{\infty} j^{-(1+\alpha)} \sum_{k=1}^{\infty} k^{-(1+\alpha)}\left(\left|D^{2} v\right|^{p_{0}}\right)_{Q_{1}\left(t_{1}-(j-1) 2^{-2 / \alpha}-k+1,0\right)}^{1 / p_{0}} \\
& =\sum_{m=1}^{\infty} \sum_{\substack{j \in \mathbb{N}, j \geq 1 \\
m-1 \leq(j-1) 2^{-2 / \alpha}<m}} j^{-(1+\alpha)} \sum_{k=1}^{\infty} k^{-(1+\alpha)}\left(\left|D^{2} v\right|^{p_{0}}\right)_{Q_{1}\left(t_{1}-(j-1) 2^{-2 / \alpha}-k+1,0\right)}^{1 / p_{0}} \\
& \leq \sum_{m=1}^{\infty} \sum_{\substack{j \in \mathbb{N}, j \geq 1 \\
m-1 \leq(j-1) 2^{-2 / \alpha}<m}} m^{-(1+\alpha)} \sum_{k=1}^{\infty} k^{-(1+\alpha)}\left(\left|D^{2} v\right|^{p_{0}}\right)_{Q_{1}\left(t_{1}-(j-1) 2^{-2 / \alpha}-k+1,0\right)}^{1 / p_{0}}
\end{aligned}
$$

where we used the inequalities

$$
m-1 \leq(j-1) 2^{-2 / \alpha}<m
$$

so that

$$
j^{-(1+\alpha)} \leq\left((m-1) 2^{2 / \alpha}+1\right)^{-(1+\alpha)} \leq m^{-(1+\alpha)} .
$$

Using (4.16) again, we see that

$$
\begin{aligned}
& \left(\left|D^{2} v\right|^{p_{0}}\right)_{Q_{1}\left(t_{1}-(j-1) 2^{-2 / \alpha}-k+1,0\right)}^{1 / p_{0}} \\
& \leq\left(\left|D^{2} v\right|^{p_{0}}\right)_{Q_{1}\left(t_{1}-m-k+1,0\right)}^{1 / p_{0}}+\left(\left|D^{2} v\right|^{p_{0}}\right)_{Q_{1}\left(t_{1}-m-k+2,0\right)}^{1 / p_{0}} .
\end{aligned}
$$

We then use the fact that for each $m$, the number of $j \in \mathbb{N}$ such that

$$
m-1 \leq(j-1) 2^{-2 / \alpha}<m
$$

is at most a fixed number determined by $\alpha$ to obtain that

$$
\begin{aligned}
& \left(\left|D^{2} v\right|^{p_{2}}\right)_{Q_{1 / 4}\left(t_{1}, 0\right)}^{1 / p_{2}} \leq N \sum_{m=1}^{\infty} m^{-(1+\alpha)} \sum_{k=1}^{\infty} k^{-(1+\alpha)}\left(\left|D^{2} v\right|^{p_{0}}\right)_{Q_{1}\left(t_{1}-m-k+1,0\right)}^{1 / p_{0}} \\
& +N \sum_{m=1}^{\infty} m^{-(1+\alpha)} \sum_{k=1}^{\infty} k^{-(1+\alpha)}\left(\left|D^{2} v\right|^{p_{0}}\right)_{Q_{1}\left(t_{1}-m-k+2,0\right)}^{1 / p_{0}}
\end{aligned}
$$


where for the first term on the right-hand side (in the same way for the second term), using Lemma 4.2 we have

$$
\begin{aligned}
& \sum_{m=1}^{\infty} m^{-(1+\alpha)} \sum_{k=1}^{\infty} k^{-(1+\alpha)}\left(\left|D^{2} v\right|^{p_{0}}\right)_{Q_{1}\left(t_{1}-m-k+1,0\right)}^{1 / p_{0}} \\
& =\sum_{m=1}^{\infty} m^{-(1+\alpha)} \sum_{k=m+1}^{\infty}(k-m)^{-(1+\alpha)}\left(\left|D^{2} v\right|^{p_{0}}\right)_{Q_{1}\left(t_{1}-k+1,0\right)}^{1 / p_{0}} \\
& =\sum_{k=2}^{\infty}\left(\left|D^{2} v\right|^{p_{0}}\right)_{Q_{1}\left(t_{1}-k+1,0\right)}^{1 / p_{0}} \sum_{m=1}^{k-1} m^{-(1+\alpha)}(k-m)^{-(1+\alpha)} \\
& \leq N \sum_{k=2}^{\infty} k^{-(1+\alpha)}\left(\left|D^{2} v\right|^{p_{0}}\right)_{Q_{1}\left(t_{1}-k+1,0\right)}^{1 / p_{0}} .
\end{aligned}
$$

Combining the above inequalities, we arrive at

$$
\left(\left|D^{2} v\right|^{p_{2}}\right)_{Q_{1 / 4}\left(t_{1}, 0\right)}^{1 / p_{2}} \leq N \sum_{k=1}^{\infty} k^{-(1+\alpha)}\left(\left|D^{2} v\right|^{p_{0}}\right)_{Q_{1}\left(t_{1}-k+1,0\right)}^{1 / p_{0}} .
$$

Repeating this procedure finite times and using a covering argument, we obtain (4.12).

The inequality (4.13) follows directly from Lemma 4.1 if $p_{0}>d / 2+1 / \alpha$. If $p_{0} \leq d / 2+1 / \alpha$, we first have (4.12) with $r=1 / 2$ for a sufficiently large $p$ so that $p \in(d / 2+1 / \alpha, \infty)$ and $v \in \mathbb{H}_{p, 0}^{\alpha, 2}\left(\left(0, t_{0}\right) \times B_{1 / 4}\right)$. Then by using (4.6) with $r=1 / 4$ and the covering argument as above we arrive at (4.13) with $r=1$. The proposition is proved.

Remark 4.4. The right-hand sides of (4.12) and (4.13) can be bounded by

$$
N\left(\mathcal{S M}\left|D^{2} v\right|^{p_{0}}\right)^{1 / p_{0}}\left(t_{0}, 0\right),
$$

provided that $\mathcal{S} \mathcal{M}\left(\left|D^{2} v\right|^{p_{0}}\right)\left(t_{0}, 0\right)$ is well defined. Indeed, thanks to scaling it is enough to check this when $r=1$. By using Hölder's inequality (or the $l_{1}$-average is less than or equal to the $l_{p_{0}}$-average),

$$
\begin{aligned}
& \sum_{j=1}^{\infty} j^{-(1+\alpha)}\left(\left|D^{2} v\right|^{p_{0}}\right)_{Q_{1}\left(t_{0}-j+1,0\right)}^{1 / p_{0}} \\
& \leq \sum_{k=0}^{\infty} \sum_{j=2^{k}}^{2^{k+1}-1} 2^{-k(1+\alpha)}\left(\left|D^{2} v\right|^{p_{0}}\right)_{Q_{1}\left(t_{0}-j+1,0\right)}^{1 / p_{0}} \\
& \leq \sum_{k=0}^{\infty} 2^{-k \alpha}\left[2^{-k} \sum_{j=2^{k}}^{2^{k+1}-1}\left(\left|D^{2} v\right|^{p_{0}}\right)_{Q_{1}\left(t_{0}-j+1,0\right)}\right]^{1 / p_{0}} \\
& =\sum_{k=0}^{\infty} 2^{-k \alpha}\left(\left|D^{2} v\right|^{p_{0}}\right)_{\left(t_{0}-2^{k+1}+1, t_{0}-2^{k}+1\right) \times B_{1}}^{1 / p_{0}} \\
& \leq \sum_{k=0}^{\infty} 2^{-k \alpha}\left(\mathcal{S M}\left|D^{2} v\right|^{p_{0}}\right)^{1 / p_{0}}\left(t_{0}, 0\right) .
\end{aligned}
$$


4.2. Estimates of $w$. Below we denote $\mathcal{H}_{2,0}^{\alpha, 1}\left((0, T) \times \mathbb{R}^{d}\right)$ to be the set of functions which can be approximated by a sequence $\left\{u_{n}\right\} \subset C_{0}^{\infty}\left([0, T] \times \mathbb{R}^{d}\right)$ with $u_{n}(0, \cdot)=$ 0 in the norm

$$
\|u\|_{\mathcal{H}_{2}^{\alpha, 1}\left((0, T) \times \mathbb{R}^{d}\right)}=\|u\|_{L_{p}\left((0, T) \times \mathbb{R}^{d}\right)}+\|D u\|_{L_{p}\left((0, T) \times \mathbb{R}^{d}\right)}+\left\|\partial_{t}^{\alpha} u\right\|_{\mathbb{H}_{p}^{-1}\left((0, T) \times \mathbb{R}^{d}\right)} .
$$

For details about $\mathcal{H}_{2,0}^{\alpha, 1}\left((0, T) \times \mathbb{R}^{d}\right)$, see 7 .

Lemma 4.5. Let $p_{0} \in(1, \infty), t_{0} \in(0, \infty), f \in L_{p_{0}}\left(\left(0, t_{0}\right) \times B_{1}\right)$, and $w \in$ $\mathcal{H}_{2,0}^{\alpha, 1}\left(\left(0, t_{0}\right) \times B_{1}\right)$ be a weak solution to (4.2) in $\left(0, t_{0}\right) \times B_{1}$ with the zero boundary condition on $\partial_{p}\left(\left(0, t_{0}\right) \times B_{1}\right)$. Then we have

$$
\|w\|_{L_{p_{0}}\left(\left(0, t_{0}\right) \times B_{1}\right)} \leq N\|f\|_{L_{p_{0}}\left(\left(0, t_{0}\right) \times B_{1}\right)},
$$

where $N=N\left(d, \delta, p_{0}\right)$ is independent of $t_{0}$.

Proof. We consider two cases.

Case 1: $p_{0} \in[2, \infty)$. For $\lambda \geq 1$, let $F(u)=|u|^{p_{0}}$ when $|u| \leq \lambda$ and $F(u)=$ $\left(p_{0} / 2\right) \lambda^{p_{0}-2}|u|^{2}$ when $|u|>\lambda$. Note that $F$ is a $C^{1}$ convex function. We multiply both sides of (4.2) by $-F^{\prime}(w)$ and integrate in $\left(0, t_{0}\right) \times B_{1}$. By noting that because $F$ is convex the integral involving $\partial_{t}^{\alpha} w$ is nonnegative (see, for example, the proof of [7, Lemma 4.1]), we have

$$
\begin{aligned}
& \int_{\left(0, t_{0}\right) \times B_{1}} a_{i j} D_{j} w D_{i} w\left(p_{0}\left(p_{0}-1\right)|w|^{p_{0}-2} \chi_{|w| \leq \lambda}+p_{0} \lambda^{p_{0}-2} \chi_{|w|>\lambda}\right) d x d t \\
& \leq \int_{\left(0, t_{0}\right) \times B_{1}}|f|\left(p_{0}|w|^{p_{0}-1} \chi_{|w| \leq \lambda}+p_{0} \lambda^{p_{0}-2}|w| \chi_{|w|>\lambda}\right) d x d t .
\end{aligned}
$$

By using the zero boundary condition and the Poincaré inequality to $G(w)$, where $G(u)=|u|^{p_{0} / 2}$ when $|u| \leq \lambda$ and $G(u)=\lambda^{p_{0} / 2-1}|u|$ when $|u|>\lambda$, we then get

$$
\begin{aligned}
& \int_{\left(0, t_{0}\right) \times B_{1}}\left(|w|^{p_{0}} \chi_{|w| \leq \lambda}+\lambda^{p_{0}-2}|w|^{2} \chi_{|w|>\lambda}\right) d x d t \\
& \quad=\int_{\left(0, t_{0}\right) \times B_{1}}|G(w)|^{2} d x d t \leq N(d) \int_{\left(0, t_{0}\right) \times B_{1}}|D(G(w))|^{2} d x d t \\
& \quad \leq N(d) \int_{\left(0, t_{0}\right) \times B_{1}}|D w|^{2}\left(\frac{p_{0}^{2}}{4}|w|^{p_{0}-2} \chi_{|w| \leq \lambda}+\lambda^{p_{0}-2} \chi_{|w|>\lambda}\right) d x d t \\
& \quad \leq N(d) \int_{\left(0, t_{0}\right) \times B_{1}}|D w|^{2}\left(p_{0}\left(p_{0}-1\right)|w|^{p_{0}-2} \chi_{|w| \leq \lambda}+p_{0} \lambda^{p_{0}-2} \chi_{|w|>\lambda}\right) d x d t \\
& \quad \leq N(d, \delta) \int_{\left(0, t_{0}\right) \times B_{1}}|f|\left(p_{0}|w|^{p_{0}-1} \chi_{|w| \leq \lambda}+p_{0} \lambda^{p_{0}-2}|w| \chi_{|w|>\lambda}\right) d x d t .
\end{aligned}
$$

From this and Young's inequality, we obtain that

$$
\begin{aligned}
& \int_{\left(0, t_{0}\right) \times B_{1}}\left|w \chi_{|w| \leq \lambda}\right|^{p_{0}}+\lambda^{p_{0}-2}\left|w \chi_{|w|>\lambda}\right|^{2} \\
& \leq N \int_{\left(0, t_{0}\right) \times B_{1}}|f|^{p_{0}} \chi_{|w| \leq \lambda}+\lambda^{p_{0}-2}|f|^{2} \chi_{|w|>\lambda},
\end{aligned}
$$

where $N=N\left(d, \delta, p_{0}\right)$. This inequality with the fact that $f \in L_{p_{0}}\left(\left(0, t_{0}\right) \times B_{1}\right)$ shows that

$$
\int_{\left(0, t_{0}\right) \times B_{1}}|w|^{p_{0}} \chi_{|w| \leq \lambda} d x d t \leq N \int_{\left(0, t_{0}\right) \times B_{1}}|f|^{p_{0}} d x d t .
$$


Taking $\lambda \rightarrow \infty$ and applying the monotone convergence theorem, we see that $w \in L_{p_{0}}\left(\left(0, t_{0}\right) \times B_{1}\right)$ and (4.18) holds.

Case 2: $p_{0} \in(1,2)$. We use a duality argument. For any $g \in C^{\infty}\left(\left(0, t_{0}\right) \times B_{1}\right)$, let $w_{1}$ be the unique $\mathcal{H}_{2,0}^{\alpha, 1}\left(\left(0, t_{0}\right) \times B_{1}\right)$ of the equation

$$
-\partial_{t}^{\alpha} w_{1}+a^{i j}\left(t_{0}-t\right) D_{i j} w_{1}=g\left(t_{0}-t, x\right)
$$

in $\left(\left(0, t_{0}\right) \times B_{1}\right)$ with the zero boundary condition on $\partial_{p}\left(\left(0, t_{0}\right) \times B_{1}\right)$. See [18] for the existence and uniqueness of solutions. From Case 1 , we have

$$
\left\|w_{1}\right\|_{L_{q_{0}}\left(\left(0, t_{0}\right) \times B_{1}\right)} \leq N\|g\|_{L_{q_{0}}\left(\left(0, t_{0}\right) \times B_{1}\right)},
$$

where $q_{0}=p_{0} /\left(p_{0}-1\right) \in(2, \infty)$. Denote $w_{2}(t, x)=w_{1}\left(t_{0}-t, x\right)$. Then by using integration by parts, we have

$$
\int_{\left(0, t_{0}\right) \times B_{1}} w g d x d t=\int_{\left(0, t_{0}\right) \times B_{1}} w_{2} f d x d t .
$$

This together with (4.19) immediately gives (4.18).

The lemma is proved.

Proposition 4.6. Let $p_{0} \in(1, \infty), f \in L_{p_{0}}\left(\left(0, t_{0}\right) \times B_{1}\right)$, and $w \in \mathcal{H}_{2,0}^{\alpha, 1}\left(\left(0, t_{0}\right) \times\right.$ $\left.B_{1}\right)$ be a weak solution to (4.2) in $\left(0, t_{0}\right) \times B_{1}$ with the zero boundary condition on $\partial_{p}\left(\left(0, t_{0}\right) \times B_{1}\right)$. Then we have

$$
\left(\left|D^{2} w\right|^{p_{0}}\right)_{Q_{1 / 2}\left(t_{1}, 0\right)}^{1 / p_{0}} \leq \sum_{k=0}^{\infty} c_{k}\left(|f|^{p_{0}}\right)_{\left(s_{k+1}, s_{k}\right) \times B_{1}}^{1 / p_{0}}
$$

for any $t_{1} \leq t_{0}$, where $s_{k}=t_{1}-2^{k}+1$ and

$$
\sum_{k=0}^{\infty} c_{k} \leq N=N\left(d, \delta, \alpha, p_{0}\right)
$$

Proof. Since the assumptions are satisfied if $t_{0}$ is replaced with $t_{1}$, we prove only the case $t_{1}=t_{0}$. By using the solvability in [6] of equations in non-divergence form in $\left(0, t_{0}\right) \times \mathbb{R}^{d}$, one can check that $w \in \mathbb{H}_{p_{0}, 0}^{\alpha, 2}\left(\left(0, t_{0}\right) \times B_{1-\varepsilon}\right)$ for any $\varepsilon \in(0,1)$. By using a cutoff function as in the proof of Lemma 4.1 (cf. (4.10) ), we have

$$
\left(\left|D^{2} w\right|^{p_{0}}\right)_{Q_{1 / 2}\left(t_{0}, 0\right)}^{1 / p_{0}} \leq N\left(|f|^{p_{0}}\right)_{Q_{1}\left(t_{0}, 0\right)}^{1 / p_{0}}+N \sum_{k=0}^{\infty} 2^{-k \alpha} A_{k}
$$

where

$$
A_{k}=\left(|w|^{p_{0}}\right)_{\left(s_{k+1}, s_{k}\right) \times B_{1}}^{1 / p_{0}}, \quad s_{k}=t_{0}-2^{k}+1 .
$$

It remains to estimate $A_{k}$ for $k=0,1, \ldots$ To this end, we take cutoff functions $\eta_{k} \in C^{\infty}(\mathbb{R})$ such that $\eta_{k}=1$ for $t \geq s_{k+1}, \eta_{k}=0$ for $t \leq s_{k+2}$, and $\left\|\eta_{k}^{\prime}\right\|_{L_{\infty}} \leq 2^{-k}$. Then $w \eta_{k}$ satisfies

$$
-\partial_{t}^{\alpha}\left(w \eta_{k}\right)+a^{i j} D_{i j}\left(w \eta_{k}\right)=g_{k}
$$

in $\left(s_{k+2}, t_{0}\right) \times B_{1}$ with the zero boundary condition on $\partial_{p}\left(\left(s_{k+2}, t_{0}\right) \times B_{1}\right)$, where $\partial_{t}^{\alpha}=\partial_{t} I_{s_{k+2}}^{1-\alpha}$ and

$$
g_{k}=f \eta_{k}-\frac{\alpha}{\Gamma(1-\alpha)} \int_{\infty}^{t}(t-s)^{-\alpha-1}\left(\eta_{k}(t)-\eta_{k}(s)\right) w(s, x) d s
$$


in $\left(s_{k+2}, t_{0}\right) \times B_{1}$. By Lemma 4.5 applied to 4.23),

$$
\int_{\left(s_{k+2}, s_{k}\right) \times B_{1}}\left|w \eta_{k}\right|^{p_{0}} \leq N \int_{\left(s_{k+2}, s_{k}\right) \times B_{1}}\left|g_{k}\right|^{p_{0}},
$$

which together with (4.24) further implies that

$$
\int_{s_{k+1}}^{s_{k}} \int_{B_{1}}|w|^{p_{0}} d x d t \leq N \int_{s_{k+2}}^{s_{k}} \int_{B_{1}}|f|^{p_{0}} d x d t+N\|J\|_{L_{p_{0}}\left(\left(s_{k+2}, s_{k}\right) \times B_{1}\right)}^{p_{0}},
$$

where $N=N\left(d, \delta, \alpha, p_{0}\right)$ and

$$
J=\int_{-\infty}^{t}(t-s)^{-\alpha-1}\left(\eta_{k}(s)-\eta_{k}(t)\right) w(s, x) d s .
$$

Note that $\eta_{k}(s)-\eta_{k}(t)=0$ for $s \in\left(s_{k+1}, t\right)$ and $t \in\left(s_{k+1}, s_{k}\right)$. Thus,

$$
\begin{aligned}
J & =\int_{-\infty}^{t}(t-s)^{-\alpha-1}\left(\eta_{k}(s)-\eta_{k}(t)\right) w(s, x) \chi_{s \leq s_{k+1}} d s \\
& =\int_{s_{k+3}}^{t}(t-s)^{-\alpha-1}\left(\eta_{k}(s)-\eta_{k}(t)\right) w(s, x) \chi_{s \leq s_{k+1}} d s \\
& +\int_{-\infty}^{s_{k+3}}(t-s)^{-\alpha-1}\left(\eta_{k}(s)-\eta_{k}(t)\right) w(s, x) d s=: J_{1}+J_{2}
\end{aligned}
$$

where $t \in\left(s_{k+2}, s_{k}\right)$ and $t-s \geq s_{k+2}-s_{k+3}=2^{k+2}$ for $s \leq s_{k+3}$. Since

$$
\left|\eta_{k}(t)-\eta_{k}(s)\right| \leq 2^{-k}|t-s|,
$$

it follows that

$$
\begin{aligned}
J_{1} & \leq 2^{-k} \int_{s_{k+3}}^{t}(t-s)^{-\alpha}|w(s, x)| \chi_{s \leq s_{k+1}} d s \\
& \leq 2^{-k} \int_{0}^{7 \cdot 2^{k}} s^{-\alpha}|w(t-s, x)| \chi_{s \geq t-s_{k+1}} d s .
\end{aligned}
$$

Using the Minkowski inequality, we have

$$
\begin{aligned}
\left\|J_{1}\right\|_{L_{p_{0}}\left(\left(s_{k+2}, s_{k}\right) \times B_{1}\right)} & \leq 2^{-k} \int_{0}^{7 \cdot 2^{k}} s^{-\alpha} \| w(\cdot-s, \cdot) \chi \cdot-s \leq s_{k+1} \\
& \leq N(\alpha) 2_{L_{p_{0}}\left(\left(s_{k+2}, s_{k}\right) \times B_{1}\right)} d s \\
& \leq N 2^{-\alpha k} \sum_{j=1}^{3}\|w\|_{L_{p_{0}}\left(\left(s_{k+4}, s_{k+1}\right) \times B_{1}\right)}\|w\|_{L_{p_{0}}\left(\left(s_{k+j+1}, s_{k+j}\right) \times B_{1}\right)} .
\end{aligned}
$$

From the fact that

$$
\left|\eta_{k}(s)-\eta_{k}(t)\right|=\left|\eta_{k}(t)\right| \leq 1
$$

for $s<s_{k+3}$, it follows that

$$
\left|J_{2}\right| \leq \int_{-\infty}^{s_{k+3}}(t-s)^{-\alpha-1}|w(s, x)| d s=\sum_{j=k+3}^{\infty} \int_{s_{j+1}}^{s_{j}}(t-s)^{-\alpha-1}|w(s, x)| d s .
$$

Since

$$
(t-s)^{-\alpha-1} \leq 2^{\alpha+1} 2^{-j(\alpha+1)}
$$


for $s \in\left(s_{j+1}, s_{j}\right)$ and $t \in\left(s_{k+2}, s_{k}\right)$ with $j \geq k+3$, we have

$$
\left|J_{2}\right| \leq 2^{\alpha+1} \sum_{j=k+3}^{\infty} 2^{-j(\alpha+1)} \int_{s_{j+1}}^{s_{j}}|w(s, x)| d s .
$$

Then by the Minkowski inequality and Hölder's inequality,

$$
\begin{gathered}
\left\|J_{2}\right\|_{L_{p_{0}}\left(\left(s_{k+2}, s_{k}\right) \times B_{1}\right)} \leq 2^{\alpha+1} \sum_{j=k+3}^{\infty} 2^{-j(\alpha+1)} \int_{s_{j+1}}^{s_{j}}\|w(s, \cdot)\|_{L_{p_{0}}\left(\left(s_{k+2}, s_{k}\right) \times B_{1}\right)} d s \\
\leq N(d) 2^{\alpha+1} \sum_{j=k+3}^{\infty} 2^{-\alpha j}\left(3 \cdot 2^{k}\right)^{1 / p_{0}}\left(f_{s_{j+1}}^{s_{j}} f_{B_{1}}|w(s, x)|^{p_{0}} d s d x\right)^{1 / p_{0}} .
\end{gathered}
$$

Combining the estimates for $J_{1}$ and $J_{2}$ with (4.25), we arrive at

$$
A_{k} \leq N\left(|f|^{p_{0}}\right)_{\left(s_{k+2}, s_{k}\right) \times B_{1}}^{1 / p_{0}}+N_{0} \sum_{j=k+1}^{\infty} 2^{-\alpha j} A_{j},
$$

where the constants $N$ and $N_{0}$ depend only on $d, \delta, \alpha$, and $p_{0}$. Now we take a large $k_{0}$ such that $N_{0} 2^{-\alpha k_{0}} /\left(1-2^{-\alpha}\right) \leq 1 / 2$. Multiplying the above inequality by $2^{-\alpha k}$ and summing in $k=k_{0}, k_{0}+1, \ldots$, we get

$$
\begin{aligned}
\sum_{k=k_{0}}^{\infty} 2^{-\alpha k} A_{k} & \leq N \sum_{k=k_{0}}^{\infty} 2^{-\alpha k}\left(|f|^{p_{0}}\right)_{\left(s_{k+2}, s_{k}\right) \times B_{1}}^{1 / p_{0}}+N_{0} \sum_{k=k_{0}}^{\infty} 2^{-\alpha k} \sum_{j=k+1}^{\infty} 2^{-\alpha j} A_{j} \\
& =N \sum_{k=k_{0}}^{\infty} 2^{-\alpha k}\left(|f|^{p_{0}}\right)_{\left(s_{k+2}, s_{k}\right) \times B_{1}}^{1 / p_{0}}+N_{0} \sum_{j=k_{0}+1}^{\infty}\left(2^{-\alpha j} A_{j} \sum_{k=k_{0}}^{j-1} 2^{-\alpha k}\right) \\
& \leq N \sum_{k=k_{0}}^{\infty} 2^{-\alpha k}\left(|f|^{p_{0}}\right)_{\left(s_{k+2}, s_{k}\right)}^{1 / p_{0}}+\frac{1}{2} \sum_{j=k_{0}+1}^{\infty} 2^{-\alpha j} A_{j} .
\end{aligned}
$$

Therefore, we have

$$
\sum_{k=k_{0}}^{\infty} 2^{-\alpha k} A_{k} \leq N \sum_{k=k_{0}}^{\infty} 2^{-\alpha k}\left(|f|^{p_{0}}\right)_{\left(s_{k+2}, s_{k}\right)}^{1 / p_{0}} .
$$

Finally, for $k=k_{0}-1, k_{0}-2, \ldots, 0$, by using (4.26) and induction, we get

$$
A_{k} \leq N \sum_{j=0}^{\infty} c_{j}\left(|f|^{p_{0}}\right)_{\left(s_{j+2}, s_{j}\right)}^{1 / p_{0}} .
$$

Combining the above inequalities with (4.22), we reach (4.20) with different $c_{k}$ 's. The proposition is proved.

\subsection{Estimates of $u$.}

Proposition 4.7. Let $p_{0} \in(1, \infty), T \in(0, \infty)$, and $u \in \mathbb{H}_{p_{0}, 0, \text { loc }}^{\alpha, 2}\left((0, T) \times \mathbb{R}^{d}\right)$ satisfy (4.1) in $(0, T) \times \mathbb{R}^{d}$. Then, for any $\left(t_{0}, x_{0}\right) \in(0, T] \times \mathbb{R}^{d}, r \in(0, \infty)$, and $\kappa \in(0,1 / 4)$, we have

$$
\begin{aligned}
& \left(\left|D^{2} u-\left(D^{2} u\right)_{Q_{\kappa r}\left(t_{0}, x_{0}\right)}\right|\right)_{Q_{\kappa r}\left(t_{0}, x_{0}\right)} \leq N \kappa^{\sigma}\left(\mathcal{S M}\left|D^{2} u\right|^{p_{0}}\right)^{1 / p_{0}}\left(t_{0}, x_{0}\right) \\
& +N \kappa^{-(d+2 / \alpha) / p_{0}} \sum_{k=0}^{\infty} c_{k}\left(|f|^{p_{0}}\right)_{\left(t_{0}-\left(2^{k+2}-2\right) r^{2 / \alpha}, t_{0}\right) \times B_{r}\left(x_{0}\right)}^{1 / p_{0}}
\end{aligned}
$$


where $N=N\left(d, \delta, \alpha, p_{0}\right), \sigma=\sigma\left(d, \alpha, p_{0}\right)$, and $\left\{c_{k}\right\}$ satisfies (4.21).

Proof. As in the parabolic case with local time derivative, thanks to translation and dilation, we assume that $x_{0}=1$ and $r=1$. We further assume that $u$ and $f$ are sufficiently smooth so that, in particular, $f \in L_{p_{0}} \cap L_{2}\left((0, T) \times B_{1}\right)$. Indeed, since $u \in \mathbb{H}_{p_{0}, 0}^{\alpha, 2}\left((0, T) \times B_{R}\right)$ for $R>0$, there is a sequence $\left\{u_{n}\right\}$ satisfying $u_{n} \in$ $C^{\infty}\left(\overline{(0, T) \times B_{1}}\right), u_{n}(0, x)=0$, and (2.2) as $n \rightarrow \infty$. Then we prove the desired estimate, more precisely, (4.29) with $u_{n}$ in place of $u$ and let $n \rightarrow \infty$.

Since $f \in L_{p_{0}} \cap L_{2}\left((0, T) \times B_{1}\right)$, one can use the results from [18 to find a weak solution $w \in \mathcal{H}_{2,0}^{\alpha, 1}\left(\left(0, t_{0}\right) \times B_{1}\right)$ satisfying (4.2) in $\left(0, t_{0}\right) \times B_{1}$ with the zero boundary condition on $\partial_{p}\left(\left(0, t_{0}\right) \times B_{1}\right)$. As in the proof of Proposition 4.6, we see that $w \in \mathbb{H}_{p_{0}, 0}^{\alpha, 2}\left(\left(0, t_{0}\right) \times B_{1-\varepsilon}\right)$ for any sufficiently small $\varepsilon>0$. Set $v=u-w$, which belongs to $\mathbb{H}_{p_{0}, 0}^{\alpha, 2}\left(\left(0, t_{0}\right) \times B_{1-\varepsilon}\right)$ and satisfies [4.3) in $\left(0, t_{0}\right) \times B_{1-\varepsilon}$. Note that because $\kappa<1 / 4$,

$$
\left(\left|D^{2} v-\left(D^{2} v\right)_{Q_{\kappa}\left(t_{0}, 0\right)}\right|\right)_{Q_{\kappa}\left(t_{0}, 0\right)} \leq N \kappa^{\sigma}\left[D^{2} v\right]_{C^{\sigma \alpha / 2, \sigma}\left(Q_{1 / 4}\left(t_{0}, 0\right)\right)} .
$$

By Proposition 4.3 with $r=1 / 2$ as well as the triangle inequality, we observe that

$$
\begin{gathered}
{\left[D^{2} v\right]_{C^{\sigma \alpha / 2, \sigma}\left(Q_{1 / 4}\left(t_{0}, 0\right)\right)} \leq N \sum_{j=1}^{\infty} j^{-(1+\alpha)}\left(\left|D^{2} v\right|^{p_{0}}\right)_{Q_{1 / 2}\left(t_{0}-(j-1) 2^{-2 / \alpha}, 0\right)}^{1 / p_{0}}} \\
\leq N \sum_{j=1}^{\infty} j^{-(1+\alpha)}\left(\left|D^{2} u\right|^{p_{0}}\right)_{Q_{1 / 2}\left(t_{0}-(j-1) 2^{-2 / \alpha}, 0\right)}^{1 / p_{0}} \\
\quad+N \sum_{j=1}^{\infty} j^{-(1+\alpha)}\left(\left|D^{2} w\right|^{p_{0}}\right)_{Q_{1 / 2}\left(t_{0}-(j-1) 2^{-2 / \alpha}, 0\right)}^{1 / p_{0}} .
\end{gathered}
$$

To estimate the summation involving $D^{2} w$ above, we first note that by (4.20), for $j=1,2, \ldots$,

$$
\left(\left|D^{2} w\right|^{p_{0}}\right)_{Q_{1 / 2}\left(t_{0}-(j-1) 2^{-2 / \alpha}, 0\right)}^{1 / p_{0}} \leq \sum_{k=0}^{\infty} c_{k}\left(|f|^{p_{0}}\right)_{\left(s_{k+1}^{j}, s_{k}^{j}\right) \times B_{1}}^{1 / p_{0}},
$$

where

$$
s_{k}^{j}=t_{0}-(j-1) 2^{-2 / \alpha}-2^{k}+1 .
$$

Then

$$
\begin{aligned}
& \sum_{j=1}^{\infty} j^{-(1+\alpha)}\left(\left|D^{2} w\right|^{p_{0}}\right)_{Q_{1 / 2}\left(t_{0}-(j-1) 2^{-2 / \alpha}, 0\right)}^{1 / p_{0}} \\
& \quad \leq \sum_{j=1}^{\infty} j^{-(1+\alpha)} \sum_{k=0}^{\infty} c_{k}\left(|f|^{p_{0}}\right)_{\left(s_{k+1}^{j}, s_{k}^{j}\right) \times B_{1}}^{1 / p_{0}} \\
& \quad=\sum_{m=1}^{\infty} \sum_{\substack{j \in \mathbb{N}, j \geq 1 \\
m-1 \leq(j-1) 2^{-2 / \alpha}<m}} j^{-(1+\alpha)} \sum_{k=0}^{\infty} c_{k}\left(|f|^{p_{0}}\right)_{\left(s_{k+1}^{j}, s_{k}^{j}\right) \times B_{1}}^{1 / p_{0}}
\end{aligned}
$$

For

it holds that

$$
m-1 \leq(j-1) 2^{-2 / \alpha}<m,
$$

$$
\left(s_{k+1}^{j}, s_{k}^{j}\right) \subset\left(t_{0}-2^{k+1}+1-m, t_{0}-m+1\right) .
$$


This shows that

$$
\left(|f|^{p_{0}}\right)_{\left(s_{k+1}^{j}, s_{k}^{j}\right) \times B_{1}}^{1 / p_{0}} \leq 2^{1 / p_{0}}\left(|f|^{p_{0}}\right)_{\left(s_{k+1}-m, t_{0}-m+1\right) \times B_{1}}^{1 / p_{0}},
$$

where $s_{k}=t_{0}-2^{k}+1$. Thus,

$$
\begin{aligned}
& \sum_{j=1}^{\infty} j^{-(1+\alpha)}\left(\left|D^{2} w\right|^{p_{0}}\right)_{Q_{1 / 2}\left(t_{0}-(j-1) 2^{-2 / \alpha}, 0\right)}^{1 / p_{0}} \\
& \leq N \sum_{m=1}^{\infty} \sum_{\substack{j \in \mathbb{N}, j \geq 1 \\
m-1 \leq(j-1) 2^{-2 / \alpha}<m}} j^{-(1+\alpha)} \sum_{k=0}^{\infty} c_{k}\left(|f|^{p_{0}}\right)_{\left(s_{k+1}-m, t_{0}-m+1\right) \times B_{1}}^{1 / p_{0}} \\
& \leq N \sum_{m=1}^{\infty} m^{-(1+\alpha)} \sum_{k=0}^{\infty} c_{k}\left(|f|^{p_{0}}\right)_{\left(s_{k+1}-m, t_{0}-m+1\right) \times B_{1}}^{1 / p_{0}} \\
& \leq N \sum_{l=0}^{\infty} \sum_{m=2^{l}}^{2^{l+1}-1} 2^{-l(1+\alpha)} \sum_{k=0}^{\infty} c_{k}\left(|f|^{p_{0}}\right)_{\left(s_{k+1}-m, t_{0}-m+1\right) \times B_{1}}^{1 / p_{0}} \\
& =N \sum_{l=0}^{\infty} 2^{-l(1+\alpha)} \sum_{k=0}^{\infty} c_{k} \sum_{m=2^{l}}^{2^{l+1}-1}\left(|f|^{p_{0}}\right)_{\left(s_{k+1}-m, t_{0}-m+1\right) \times B_{1}}^{1 / p_{0}} \\
& \leq N \sum_{l=0}^{\infty} 2^{-l \alpha} \sum_{k=0}^{\infty} c_{k}\left[\sum_{m=2^{l}}^{2^{l+1}-1} 2^{-l}\left(|f|^{p_{0}}\right)_{\left(s_{k+1}-m, t_{0}-m+1\right) \times B_{1}}\right.
\end{aligned}
$$

where in the last inequality we used Hölder's inequality. We further observe that

$$
\begin{aligned}
& \sum_{m=2^{l}}^{2^{l+1}-1} 2^{-l}\left(|f|^{p_{0}}\right)_{\left(s_{k+1}-m, t_{0}-m+1\right) \times B_{1}} \\
& =2^{-l} 2^{-(k+1)} \sum_{m=2^{l}}^{2^{l+1}-1} \int_{s_{k+1}-m}^{t_{0}-m+1} f_{B_{1}}|f|^{p_{0}} d x d t \\
& =2^{-l} 2^{-(k+1)} \sum_{m=2^{l}}^{2^{l+1}-1} \sum_{i=0}^{2^{k+1}-1}\left(|f|^{p_{0}}\right)_{Q_{1}\left(t_{0}-m-i+1,0\right)} \\
& \leq 2^{-l-k} 2^{\min \{l, k\}} \sum_{m=2^{l}}^{2^{l+1}+2^{k+1}-2}\left(|f|^{p_{0}}\right)_{Q_{1}\left(t_{0}-m+1,0\right)} \\
& \leq\left\{\begin{array}{lll}
4\left(|f|^{p_{0}}\right)_{\left(t_{0}-2^{l+2}+2, t_{0}\right) \times B_{1}} & \text { for } \quad l \geq k, \\
4\left(|f|^{p_{0}}\right)_{\left(t_{0}-2^{k+2}+2, t_{0}\right) \times B_{1}} & \text { for } \quad k>l .
\end{array}\right.
\end{aligned}
$$

From the above two sets of estimates it follows that

$$
\sum_{j=1}^{\infty} j^{-(1+\alpha)}\left(\left|D^{2} w\right|^{p_{0}}\right)_{Q_{1 / 2}\left(t_{0}-(j-1) 2^{-2 / \alpha}, 0\right)} \leq \sum_{k=0}^{\infty} c_{k}\left(|f|^{p_{0}}\right)_{\left(t_{0}-2^{k+2}+2, t_{0}\right) \times B_{1}}^{1 / p_{0}},
$$


where $\left\{c_{k}\right\}$ is another sequence satisfying (4.21). This combined with (4.27) and (4.28) shows that

$$
\begin{aligned}
\left(\left|D^{2} v-\left(D^{2} v\right)_{Q_{\kappa}\left(t_{0}, 0\right)}\right|\right)_{Q_{\kappa}\left(t_{0}, 0\right)} \leq & N \kappa^{\sigma} \sum_{j=1}^{\infty} j^{-(1+\alpha)}\left(\left|D^{2} u\right|^{p_{0}}\right)_{Q_{1 / 2}\left(t_{0}-(j-1) 2^{-2 / \alpha}, 0\right)}^{1 / p_{0}} \\
& +N \kappa^{\sigma} \sum_{k=0}^{\infty} c_{k}\left(|f|^{p_{0}}\right)_{\left(t_{0}-2^{k+2}+2, t_{0}\right) \times B_{1}}^{1 / p_{0}} .
\end{aligned}
$$

We then have

$$
\begin{aligned}
& \left(\left|D^{2} u-\left(D^{2} u\right)_{Q_{\kappa}\left(t_{0}, 0\right)}\right|\right)_{Q_{\kappa}\left(t_{0}, 0\right)} \\
& \leq\left(\left|D^{2} v-\left(D^{2} v\right)_{Q_{\kappa}\left(t_{0}, 0\right)}\right|\right)_{Q_{\kappa}\left(t_{0}, 0\right)}+N \kappa^{-(d+2 / \alpha) / p_{0}}\left(\left|D^{2} w\right|\right)_{Q_{1 / 2}\left(t_{0}, 0\right)} \\
& \leq \kappa^{\sigma} \sum_{j=1}^{\infty} j^{-(1+\alpha)}\left(\left|D^{2} u\right|^{p_{0}}\right)_{Q_{1 / 2}\left(t_{0}-(j-1) 2^{-2 / \alpha}, 0\right)}^{1 / p_{0}} \\
& \quad+N \kappa^{-(d+2 / \alpha) / p_{0}} \sum_{k=0}^{\infty} c_{k}\left(|f|^{p_{0}}\right)_{\left(t_{0}-2^{k+2}+2, t_{0}\right) \times B_{1}}^{1 / p_{0}}
\end{aligned}
$$

where $N=N\left(d, \delta, \alpha, p_{0}\right)$ and we used in the last inequality (4.20) along with the observation that

$$
\left(|f|^{p_{0}}\right)_{\left(t_{0}-2^{k+1}+1, t_{0}-2^{k}+1\right) \times B_{1}}^{1 / p_{0}} \leq N\left(p_{0}\right)\left(|f|^{p_{0}}\right)_{\left(t_{0}-2^{k+2}+2, t_{0}\right) \times B_{1}}^{1 / p_{0}} .
$$

Finally, we use (4.17) with a scaling and $u$ in place of $v$ to get

$$
\sum_{j=1}^{\infty} j^{-(1+\alpha)}\left(\left|D^{2} u\right|^{p_{0}}\right)_{Q_{1 / 2}\left(t_{0}-(j-1) 2^{-2 / \alpha}, 0\right)}^{1 / p_{0}} \leq N\left(\mathcal{S M}\left|D^{2} u\right|^{p_{0}}\right)^{1 / p_{0}}\left(t_{0}, 0\right)
$$

where $N=N(\alpha)$. The proposition is proved.

\section{Weight MiXeD-NORM ESTIMATES}

In this section we prove Theorem 2.2 .

Lemma 5.1. Let $\alpha \in(0,1], T \in(0, \infty), p, q \in(1, \infty), K_{1} \in[1, \infty), w=$ $w_{1}(t) w_{2}(x)$, where

$$
w_{1}(t) \in A_{p}(\mathbb{R}, d t), \quad w_{2}(x) \in A_{q}\left(\mathbb{R}^{d}, d x\right), \quad\left[w_{1}\right]_{A_{p}} \leq K_{1}, \quad\left[w_{2}\right]_{A_{q}} \leq K_{1} .
$$

There exist $p_{0}=p_{0}\left(d, p, q, K_{1}\right) \in(1, \infty)$ and $\mu=\mu\left(d, p, q, K_{1}\right) \in(1, \infty)$ such that

$$
p_{0}<p_{0} \mu<\min \{p, q\}
$$

and the following holds.

If $u \in \mathbb{H}_{p, q, w, 0}^{\alpha, 2}\left((0, T) \times \mathbb{R}^{d}\right)$ has compact support in $[0, T] \times B_{R_{0}}$ and satisfies

$$
-\partial_{t}^{\alpha} u+a^{i j}(t, x) D_{i j} u=f
$$

in $(0, T) \times \mathbb{R}^{d}$, where the coefficients a $a^{i j}(t, x)$ satisfy Assumption 2.1 $\left(\gamma_{0}\right)$, then for any $\left(t_{0}, x_{0}\right) \in(0, T] \times \mathbb{R}^{d}, r \in(0, \infty), \kappa \in(0,1 / 4)$, we have

$$
\begin{aligned}
\left(\mid D^{2} u-\right. & \left.\left(D^{2} u\right)_{Q_{\kappa r}\left(t_{0}, x_{0}\right)} \mid\right)_{Q_{\kappa r}\left(t_{0}, x_{0}\right)} \leq N \kappa^{\sigma}\left(\mathcal{S M}\left|D^{2} u\right|^{p_{0}}\right)^{1 / p_{0}}\left(t_{0}, x_{0}\right) \\
& +N \kappa^{-(d+2 / \alpha) / p_{0}} \gamma_{0}^{1 / \nu p_{0}}\left(\mathcal{S} \mathcal{M}\left|D^{2} u\right|^{\mu p_{0}}\right)^{1 / \mu p_{0}}\left(t_{0}, x_{0}\right) \\
& +N \kappa^{-(d+2 / \alpha) / p_{0}}\left(\mathcal{S} \mathcal{M}|f|^{p_{0}}\right)^{1 / p_{0}}\left(t_{0}, x_{0}\right)
\end{aligned}
$$


where $\nu=\mu /(\mu-1), \gamma=\gamma\left(d, \alpha, p_{0}\right)$, and $N=N\left(d, \delta, \alpha, p, q, K_{1}\right)$. The functions $u$ and $f$ are defined to be zero whenever $t \leq 0$.

Proof. For the given $w_{1} \in A_{p}(\mathbb{R}, d t)$ and $w_{2} \in A_{q}\left(\mathbb{R}^{d}, d x\right)$, using the reverse Hölder's inequality for $A_{p}$ weights, we find

$$
\sigma_{1}=\sigma_{1}\left(d, p, K_{1}\right), \quad \sigma_{2}=\sigma_{2}\left(d, q, K_{1}\right)
$$

such that $p-\sigma_{1}>1, q-\sigma_{2}>1$, and

$$
w_{1} \in A_{p-\sigma_{1}}(\mathbb{R}, d t), \quad w_{2} \in A_{q-\sigma_{2}}\left(\mathbb{R}^{d}, d x\right) .
$$

Set $p_{0}, \mu \in(1, \infty)$ so that

$$
p_{0} \mu=\min \left\{\frac{p}{p-\sigma_{1}}, \frac{q}{q-\sigma_{2}}\right\}>1 .
$$

Note that

$$
\begin{gathered}
w_{1} \in A_{p-\sigma_{1}} \subset A_{\frac{p}{p_{0} \mu}} \subset A_{\frac{p}{p_{0}}}(\mathbb{R}, d t), \\
w_{2} \in A_{q-\sigma_{2}} \subset A_{\frac{q}{p_{0} \mu}} \subset A_{\frac{q}{p_{0}}}\left(\mathbb{R}^{d}, d x\right) .
\end{gathered}
$$

From these inclusions and the fact that $u \in \mathbb{H}_{p, q, w, 0}^{\alpha, 2}\left((0, T) \times \mathbb{R}^{d}\right)$ it follows that (see the proof of [5, Lemma 5.10])

$$
u \in \mathbb{H}_{p_{0} \mu, 0, \text { loc }}^{\alpha, 2}\left((0, T) \times \mathbb{R}^{d}\right) .
$$

In particular, if $\alpha=1$, by extending $u$ as zero for $t<0$, we see that

$$
u \in W_{p_{0} \mu, \text { loc }}^{1,2}\left((-\infty, T) \times \mathbb{R}^{d}\right) \text {. }
$$

Set

$$
\bar{a}^{i j}(t)=\left\{\begin{array}{ccc}
f_{B_{r}\left(x_{0}\right)} a^{i j}(t, y) d y & \text { if } \quad r \leq R_{0}, \\
f_{B_{R_{0}}} a^{i j}(t, y) d y & \text { if } \quad r>R_{0},
\end{array}\right.
$$

and write

$$
-\partial_{t}^{\alpha} u+\bar{a}^{i j}(t) D_{i j} u=\tilde{f}
$$

where

$$
\tilde{f}=f+\left(\bar{a}^{i j}(t)-a^{i j}(t, x)\right) D_{i j} u .
$$

Then by Propositions 3.4 and 4.7 it follows that

$$
\begin{aligned}
&\left(\left|D^{2} u-\left(D^{2} u\right)_{Q_{\kappa r}\left(t_{0}, x_{0}\right)}\right|\right)_{Q_{\kappa r}\left(t_{0}, x_{0}\right)} \leq N \kappa^{\sigma}\left(\mathcal{S M}\left|D^{2} u\right|^{p_{0}}\right)^{1 / p_{0}}\left(t_{0}, x_{0}\right) \\
&+N \kappa^{-(d+2 / \alpha) / p_{0}} \sum_{k=0}^{\infty} c_{k}\left(|\tilde{f}|^{p_{0}}\right)_{\left(t_{0}-\left(2^{k+2}-2\right) r^{2 / \alpha}, t_{0}\right) \times B_{r}\left(x_{0}\right)}^{1 / p_{0}},
\end{aligned}
$$

where $\left\{c_{k}\right\}$ satisfies (3.5) or (4.21) and $N=N\left(d, \delta, \alpha, p_{0}\right)=N\left(d, \delta, \alpha, p, q, K_{1}\right)$. Using the fact that $u$ has compact support in $[0, T] \times B_{R_{0}}$ and Hölder's inequality, we write

$$
\begin{gathered}
\left(|\tilde{f}|^{p_{0}}\right)_{\left(t_{0}-\left(2^{k+2}-2\right) r^{2 / \alpha}, t_{0}\right) \times B_{r}\left(x_{0}\right)}^{1 / p_{0}} \leq\left(|f|^{p_{0}}\right)_{\left(t_{0}-\left(2^{k+2}-2\right) r^{2 / \alpha}, t_{0}\right) \times B_{r}\left(x_{0}\right)}^{1 / p_{0}} \\
+\left(\left|\bar{a}^{i j}-a^{i j}\right|^{\nu p_{0}} \chi_{B_{R_{0}}}\right)_{\left(t_{0}-\left(2^{k+2}-2\right) r^{2 / \alpha}, t_{0}\right) \times B_{r}\left(x_{0}\right)}^{1 / \nu p_{0}} \\
\cdot\left(\left|D^{2} u\right|^{\mu p_{0}}\right)_{\left(t_{0}-\left(2^{k+2}-2\right) r^{2 / \alpha}, t_{0}\right) \times B_{r}\left(x_{0}\right)}^{1 / \mu p_{0}}
\end{gathered}
$$


where by Assumption 2.1 and the boundedness of $a^{i j}$ (also see Remark 2.3 in [6]), for $r \leq R_{0}$,

$$
\begin{aligned}
& \left(\left|\bar{a}^{i j}-a^{i j}\right|^{\nu p_{0}} \chi_{B_{R_{0}}}\right)_{\left(t_{0}-\left(2^{k+2}-2\right) r^{2 / \alpha}, t_{0}\right) \times B_{r}\left(x_{0}\right)} \\
& \leq\left(\left|\bar{a}^{i j}-a^{i j}\right|^{\nu p_{0}}\right)_{\left(t_{0}-\left(2^{k+2}-2\right) r^{2 / \alpha}, t_{0}\right) \times B_{r}\left(x_{0}\right)} \leq N \gamma_{0},
\end{aligned}
$$

and, for $r>R_{0}$,

$$
\begin{aligned}
& \left(\left|\bar{a}^{i j}-a^{i j}\right|^{\nu p_{0}} \chi_{B_{R_{0}}}\right)_{\left(t_{0}-\left(2^{k+2}-2\right) r^{2 / \alpha}, t_{0}\right) \times B_{r}\left(x_{0}\right)} \\
& \leq\left(\left|\bar{a}^{i j}-a^{i j}\right|^{\nu p_{0}}\right)_{\left(t_{0}-\left(2^{k+2}-2\right) r^{2 / \alpha}, t_{0}\right) \times B_{R_{0}}} \leq N \gamma_{0} .
\end{aligned}
$$

Thus,

$$
\begin{aligned}
& \sum_{k=0}^{\infty} c_{k}\left(|\tilde{f}|^{p_{0}}\right)_{\left(t_{0}-\left(2^{k+2}-2\right) r^{2 / \alpha}, t_{0}\right) \times B_{r}\left(x_{0}\right)}^{1 / p_{0}} \leq \sum_{k=0}^{\infty} c_{k}\left(|f|^{p_{0}}\right)^{1 / p_{0}}\left(t_{0}-\left(2^{k+2}-2\right) r^{2 / \alpha}, t_{0}\right) \times B_{r}\left(x_{0}\right) \\
& \quad+N \gamma_{0}^{1 / \nu p_{0}} \sum_{k=0}^{\infty} c_{k}\left(\left|D^{2} u\right|^{\mu p_{0}}\right)_{\left(t_{0}-\left(2^{k+2}-2\right) r^{2 / \alpha}, t_{0}\right) \times B_{r}\left(x_{0}\right)}^{1 / \mu p_{0}} \\
& \leq N\left(\mathcal{S M}|f|^{p_{0}}\right)^{1 / p_{0}}\left(t_{0}, x_{0}\right)+N \gamma_{0}^{1 / \nu p_{0}}\left(\mathcal{S M}\left|D^{2} u\right|^{\mu p_{0}}\right)^{1 / \mu p_{0}}\left(t_{0}, x_{0}\right) .
\end{aligned}
$$

This inequality together with (5.3) proves (5.2). The lemma is proved.

For each integer $n \in \mathbb{Z}$, find an integer $k(n)$ such that

$$
k(n) \leq \frac{2}{\alpha} n<k(n)+1 .
$$

Note that $k(n+1)-k(n)$ is a non-negative integer and

$$
\frac{1}{2^{k(n)+1}}<\frac{1}{2^{2 n / \alpha}} \leq \frac{1}{2^{k(n)}}
$$

Let

$$
\mathbb{C}_{n}:=\left\{Q_{\vec{i}}^{n}=Q_{\left(i_{0}, i_{1}, \ldots, i_{d}\right)}^{n}: \vec{i}=\left(i_{0}, i_{1}, \ldots, i_{d}\right) \in \mathbb{Z}^{d+1}\right\},
$$

where $n \in \mathbb{Z}$ and

$$
Q_{\vec{i}}^{n}=\left[\frac{i_{0}}{2^{k(n)}}+T, \frac{i_{0}+1}{2^{k(n)}}+T\right) \times\left[\frac{i_{1}}{2^{n}}, \frac{i_{1}+1}{2^{n}}\right) \times \cdots \times\left[\frac{i_{d}}{2^{n}}, \frac{i_{d}+1}{2^{n}}\right) .
$$

Note that $\left\{\mathbb{C}_{n}\right\}_{n \in \mathbb{Z}}$ is a collection of partitions of $\mathbb{R}^{d+1}$ satisfying [5, Theorem 2.1] with respect to the parabolic distance

$$
|(t, x)-(s, y)|:=\max \left\{|x-y|,|t-s|^{\alpha / 2}\right\} .
$$

In particular, for each $n \in \mathbb{Z}, Q_{\vec{i}}^{n}$ belongs to either $(-\infty, T) \times \mathbb{R}^{d}$ or $[T, \infty) \times \mathbb{R}^{d}$. Also note that, for each $Q_{\vec{i}}^{n}$, where $Q_{\vec{i}}^{n} \cap\left((-\infty, T) \times \mathbb{R}^{d}\right) \neq \emptyset$, there exists $Q_{r}\left(t_{0}, x_{0}\right)$ such that $t_{0} \in(-\infty, T]$ and

$$
Q_{\vec{i}}^{n} \subset Q_{r}\left(t_{0}, x_{0}\right), \quad\left|Q_{r}\left(t_{0}, x_{0}\right)\right| \leq N\left|Q_{\vec{i}}^{n}\right|,
$$

where $N=N(d, \alpha)$. Indeed, we can take, for example,

$$
t_{0}=\frac{i_{0}+1}{2^{k(n)}}+T, \quad x_{0}=\left(x_{01}, \ldots, x_{0 d}\right), \quad x_{0 j}=\frac{2 i_{l}+1}{2^{n+1}},
$$

and

$$
r=\max \left\{\sqrt{d} / 2,2^{\alpha / 2}\right\} 2^{-n} .
$$


Denote the dyadic sharp function of $g$ by

$$
g_{\mathrm{dy}}^{\#}(t, x)=\sup _{n<\infty} f_{Q_{\vec{i}}^{n} \ni(t, x)}\left|g(s, y)-g_{\mid n}(t, x)\right| d y d s,
$$

where

$$
g_{\mid n}(t, x)=f_{Q_{\vec{i}}^{n}} g(s, y) d y d s, \quad(t, x) \in Q_{\vec{i}}^{n} .
$$

Theorem 5.2 (Maximal function theorem for strong maximal functions). Let $p, q \in$ $(1, \infty), K_{1} \in[1, \infty), w_{1}(t) \in A_{p}(\mathbb{R}, d t), w_{2}(x) \in A_{q}(\mathbb{R}, d x),\left[w_{1}\right]_{A_{p}} \leq K_{1},\left[w_{2}\right]_{A_{q}} \leq$ $K_{1}$, and $w(t, x)=w_{1}(t) w_{2}(x)$. Then, for any $f \in L_{p, q, w}\left(\mathbb{R} \times \mathbb{R}^{d}\right)$, we have

$$
\|\mathcal{S M f}\|_{L_{p, q, w}\left(\mathbb{R} \times \mathbb{R}^{d}\right)} \leq N\|f\|_{L_{p, q, w}\left(\mathbb{R} \times \mathbb{R}^{d}\right)},
$$

where $N=N\left(d, p, q, K_{1}\right)>0$.

Proof. When $p=q$, this follows from [1, Theorem 1.1]. The general case is a consequence of the extrapolation theorem of Rubio de Francia [16. See also [5], Theorem 2.5].

Lemma 5.3. Let $\alpha \in(0,1], T \in(0, \infty), p, q \in(1, \infty), K_{1} \in[1, \infty), w=$ $w_{1}(t) w_{2}(x)$, where

$$
w_{1}(t) \in A_{p}(\mathbb{R}, d t), \quad w_{2}(x) \in A_{q}\left(\mathbb{R}^{d}, d x\right), \quad\left[w_{1}\right]_{A_{p}} \leq K_{1}, \quad\left[w_{2}\right]_{A_{q}} \leq K_{1} .
$$

There exists $\gamma_{0}=\gamma_{0}\left(d, \delta, \alpha, p, q, K_{1}\right)>0$ such that, under Assumption 2.1 ( $\left.\gamma_{0}\right)$, for any $u \in \mathbb{H}_{p, q, w, 0}^{\alpha, 2}\left((0, T) \times \mathbb{R}^{d}\right)$ with compact support in $[0, T] \times B_{R_{0}}$ satisfying (5.1) in $(0, T) \times \mathbb{R}^{d}$, we have

$$
\left\|\partial_{t}^{\alpha} u\right\|_{L_{p, q, w}\left((0, T) \times \mathbb{R}^{d}\right)}+\left\|D^{2} u\right\|_{L_{p, q, w}\left((0, T) \times \mathbb{R}^{d}\right)} \leq N\|f\|_{L_{p, q, w}\left((0, T) \times \mathbb{R}^{d}\right)},
$$

where $N=N\left(d, \delta, \alpha, p, q, K_{1}\right)$.

Proof. By using the partitions $\mathbb{C}_{n}$ and the dyadic sharp function introduced above, from (5.2) we obtain that

$$
\begin{aligned}
\left(D^{2} u\right)_{\mathrm{dy}}^{\#}\left(t_{0}, x_{0}\right) & \leq N \kappa^{\sigma}\left(\mathcal{S} \mathcal{M}\left|D^{2} u\right|^{p_{0}}\right)^{1 / p_{0}}\left(t_{0}, x_{0}\right) \\
& +N \kappa^{-(d+2 / \alpha) / p_{0}} \gamma_{0}^{1 / \nu p_{0}}\left(\mathcal{S} \mathcal{M}\left|D^{2} u\right|^{\mu p_{0}}\right)^{1 / \mu p_{0}}\left(t_{0}, x_{0}\right) \\
& +N \kappa^{-(d+2 / \alpha) / p_{0}}\left(\mathcal{S} \mathcal{M}|f|^{p_{0}}\right)^{1 / p_{0}}\left(t_{0}, x_{0}\right)
\end{aligned}
$$

for any $\left(t_{0}, x_{0}\right) \in \mathbb{R} \times \mathbb{R}^{d}$, provided that $D^{2} u$ is defined to be zero on $(T, \infty) \times \mathbb{R}^{d}$. Indeed, for $\left(t_{0}, x_{0}\right) \in(T, \infty) \times \mathbb{R}^{d}$, we see that

$$
\left(D^{2} u\right)_{\mathrm{dy}}^{\#}\left(t_{0}, x_{0}\right)=0
$$

by the choice of the partitions. Then by the sharp function theorem (see [5, Corollary 2.7]) and Theorem 5.2 we get

$$
\begin{aligned}
\left\|D^{2} u\right\|_{p, q, w} \leq & N\left(\kappa^{\sigma}+\kappa^{-(d+2 / \alpha) / p_{0}} \gamma_{0}^{1 / \nu p_{0}}\right)\left\|D^{2} u\right\|_{p, q, w} \\
& +N \kappa^{-(d+2 / \alpha) / p_{0}}\|f\|_{p, q, w},
\end{aligned}
$$

where $\|\cdot\|_{p, q, w}=\|\cdot\|_{L_{p, q, w}\left((0, T) \times \mathbb{R}^{d}\right)}$ and $N=N\left(d, \delta, \alpha, p, q, K_{1}\right)$. Now by first taking a sufficiently small $\kappa<1 / 4$, then taking small $\gamma_{0}$ so that

$$
\kappa^{\sigma}+\kappa^{-(d+2 / \alpha) / p_{0}} \gamma_{0}^{1 / \nu p_{0}}<\frac{1}{2 N},
$$


we arrive at

$$
\left\|D^{2} u\right\|_{L_{p, q, w}\left((0, T) \times \mathbb{R}^{d}\right)} \leq N\|f\|_{L_{p, q, w}\left((0, T) \times \mathbb{R}^{d}\right)} .
$$

Then, using this estimate and the equation, we obtain (5.4). The lemma is proved.

Corollary 5.4. Let $\alpha \in(0,1], T \in(0, \infty), p \in(1, \infty), K_{1} \in[1, \infty), w=$ $w_{1}(t) w_{2}(x)$, where

$$
w_{1}(t) \in A_{p}(\mathbb{R}, d t), \quad w_{2}(x) \in A_{q}\left(\mathbb{R}^{d}, d x\right), \quad\left[w_{1}\right]_{A_{p}} \leq K_{1}, \quad\left[w_{2}\right]_{A_{q}} \leq K_{1} .
$$

There exists $\gamma_{0}=\gamma_{0}\left(d, \delta, \alpha, p, q, K_{1}\right)>0$ such that, under Assumption 2.1 $\left(\gamma_{0}\right)$, for any $u \in \mathbb{H}_{p, q, w, 0}^{\alpha, 2}\left((0, T) \times \mathbb{R}^{d}\right)$ satisfying (2.4) in $(0, T) \times \mathbb{R}^{d}$, we have

$$
\left\|\partial_{t}^{\alpha} u\right\|_{p, q, w}+\left\|D^{2} u\right\|_{p, q, w} \leq N_{0}\|f\|_{p, q, w}+N_{1}\|u\|_{p, q, w}
$$

where $N_{0}=N_{0}\left(d, \delta, \alpha, p, q, K_{1}\right), N_{1}=N_{1}\left(d, \delta, \alpha, p, q, K_{1}, K_{0}, R_{0}\right)$, and $\|\cdot\|_{p, q, w}=$ $\|\cdot\|_{L_{p, q, w}\left((0, T) \times \mathbb{R}^{d}\right)} \cdot$

Proof. We first consider the case $p=q$. Write

$$
-\partial_{t}^{\alpha} u+a^{i j} D_{i j} u=f-b^{i} D_{i} u-c u .
$$

Then using Lemma 5.3 with $p=q$, and using the partition of unity with respect to the spatial variables, we have

$$
\left\|\partial_{t}^{\alpha} u\right\|_{p, w}+\left\|D^{2} u\right\|_{p, w} \leq N_{0}\|f\|_{p, w}+N_{1}\|D u\|_{p, w}+N_{1}\|u\|_{p, w},
$$

where $N_{0}=N_{0}\left(d, \delta, \alpha, p, q, K_{1}\right), N_{1}=N_{1}\left(d, \delta, \alpha, p, q, K_{1}, K_{0}, R_{0}\right)$, and \|\|$_{p, w}=$ $\|\cdot\|_{L_{p, w}\left((0, T) \times \mathbb{R}^{d}\right)}$. Then we use an interpolation inequality (see [8, Lemma 3.5 (iii)]) to derive (5.5) for $p=q$.

For $p \neq q$, we use the extrapolation theorem. See [5, Theorem 2.5].

To estimate $\|u\|_{L_{p, q, w}\left((0, T) \times \mathbb{R}^{d}\right)}$ on the right-hand side of (5.5), we need the following observation.

Lemma 5.5. Let $\alpha \in(0,1), p, q \in(1, \infty)$ and $K_{1} \in[1, \infty), w(t, x)=w_{1}(t) w_{2}(x)$, where

$$
w_{1} \in A_{p}(\mathbb{R}, d t), \quad w_{2} \in A_{p}\left(\mathbb{R}^{d}, d x\right), \quad\left[w_{1}\right]_{A_{p}} \leq K_{1}, \quad\left[w_{2}\right]_{A_{q}} \leq K_{1} .
$$

(a) For any $f \in L_{p, w_{1}}((0, T))$, we have

$$
\left\|I^{\alpha} f\right\|_{L_{p, w_{1}}((0, T))} \leq N T^{\alpha}\|f\|_{L_{p, w_{1}}((0, T))},
$$

where $N>0$ depends only on $\alpha, p$, and $K_{1}$.

(b) For any $g \in L_{p, q, w}\left((0, T) \times \mathbb{R}^{d}\right)$, we have

$$
\left\|I^{\alpha} g\right\|_{L_{p, q, w}\left((0, T) \times \mathbb{R}^{d}\right)} \leq N T^{\alpha}\|g\|_{L_{p, q, w}\left((0, T) \times \mathbb{R}^{d}\right)},
$$

where $N>0$ depends only on $\alpha, p, q, K_{1}$.

Proof. By scaling, we may assume that $T=1$. Indeed, when scaling, recall that $\left[w_{1}(T t)\right]_{A_{p}}=\left[w_{1}(t)\right]_{A_{p}}$. We extend $f$ to be zero when $t \notin(0, T)$. Set

$$
F(s)=\int_{0}^{s}|f(t-r)| d r
$$

which is absolutely continuous. Then, for $t \in[0,1]$,

$$
\left|I^{\alpha} f(t)\right| \leq \frac{1}{\Gamma(\alpha)} \int_{0}^{1} s^{\alpha-1}|f(t-s)| d s=\frac{1}{\Gamma(\alpha)} \int_{0}^{1} s^{\alpha-1} F^{\prime}(s) d s,
$$


where

$$
\begin{aligned}
& \int_{0}^{1} s^{\alpha-1} F^{\prime}(s) d s=\lim _{\varepsilon \searrow 0} \int_{\varepsilon}^{1} s^{\alpha-1} F^{\prime}(s) d s \\
& =\lim _{\varepsilon \searrow 0}\left(F(1)-\varepsilon^{\alpha-1} F(\varepsilon)+(1-\alpha) \int_{\varepsilon}^{1} s^{\alpha-2} F(s) d s\right) .
\end{aligned}
$$

Since

$$
s^{-1} F(s) \leq 2 \mathcal{M} f(t)
$$

for any $s \in(0,1]$, where

$$
\mathcal{M} f(t)=\frac{1}{2 r} \int_{t-r}^{t+r}|f(s)| d s
$$

we see that

$$
\int_{0}^{1} s^{\alpha-1} F^{\prime}(s) d s \leq N \mathcal{M} f(t), \quad \text { i.e., } \quad\left|I^{\alpha} f(t)\right| \leq N \mathcal{M} f(t),
$$

where $N=N(\alpha)$. Then (5.6) follows from the Hardy-Littlewood maximal function theorem with $A_{p}$ weights. Finally, we get (5.7) by using the Fubini theorem and (5.6) when $p=q$, and the general case by using the extrapolation theorem. See [5], Theorem 2.5]. The lemma is proved.

Lemma 5.6. Let $\alpha \in(0,1], T \in(0, \infty), p \in(1, \infty), K_{1} \in[1, \infty), w=w_{1}(t) w_{2}(x)$, where

$$
w_{1}(t) \in A_{p}(\mathbb{R}, d t), \quad w_{2}(x) \in A_{p}\left(\mathbb{R}^{d}, d x\right), \quad\left[w_{1}\right]_{A_{p}} \leq K_{1}, \quad\left[w_{2}\right]_{A_{p}} \leq K_{2} .
$$

For $u \in \mathbb{H}_{p, q, w, 0}^{\alpha, 2}\left((0, T) \times \mathbb{R}^{d}\right)$, we have

$$
\|u\|_{p, q, w} \leq N T^{\alpha}\left\|\partial_{t}^{\alpha} u\right\|_{p, q, w}
$$

where $N=N\left(\alpha, p, q, K_{1}\right)$ and $\|\cdot\|_{p, q, w}=\|\cdot\|_{L_{p, q, w}\left((0, T) \times \mathbb{R}^{d}\right)}$.

Proof. We first consider the case $\alpha \in(0,1)$. Since $u \in \mathbb{H}_{p, q, w, 0}^{\alpha, 2}\left((0, T) \times \mathbb{R}^{d}\right)$, we further assume that $u \in C_{0}^{\infty}\left([0, T] \times \mathbb{R}^{d}\right)$ with $u(0, \cdot)=0$. Then by [․ Lemma A.4] and Lemma [5.5

$$
\|u\|_{p, q, w}=\left\|I^{\alpha} \partial_{t}^{\alpha} u\right\|_{p, q, w} \leq N T^{\alpha}\left\|\partial_{t}^{\alpha} u\right\|_{p, q, w},
$$

where $N=N\left(\alpha, p, q, K_{1}\right)$.

For $\alpha=1$, since

$$
|u(t, x)| \leq \int_{0}^{t}\left|u_{t}(s, x)\right| d s \leq 2 t \mathcal{M} u_{t}(t, x) \leq 2 T \mathcal{M} u_{t}(t, x),
$$

the desired inequality follows as in the proof of Lemma 5.5.

We are now ready to present the proof of Theorem 2.2

Proof of Theorem 2.2. We first prove the estimate (2.5). We only consider $\alpha \in$ $(0,1)$ because the case $\alpha=1$ is simpler. By Corollary 5.4 and the interpolation inequality used in the proof of Corollary 5.4 it suffices to show that

$$
\|u\|_{p, q, w} \leq N\|f\|_{p, q, w}
$$

By extending $u$ and $f$ as zero for $t<0$, we observe (see [6, Lemma 3.5]) that

$$
-\partial_{t}^{\alpha} u+a^{i j}(t, x) D_{i j} u+b^{i} D_{i} u+c u=f
$$


in $(S, T) \times \mathbb{R}^{d}$ for any $S \leq 0$, where

$$
\partial_{t}^{\alpha} u=\partial_{t} I_{S}^{1-\alpha} u
$$

Take a positive integer $m$ to be specified below and set

$$
s_{j}=\frac{j T}{m}, \quad j=-1,0,1,2, \ldots, m
$$

We then take cutoff functions $\eta_{j} \in C^{\infty}(\mathbb{R}), j=0,1,2, \ldots, m-1$, such that

$$
\eta_{j}=\left\{\begin{array}{ll}
1 & \text { for } \quad t \geq s_{j}, \\
0 & \text { for } \quad t \leq s_{j-1},
\end{array} \quad\left|\eta_{j}^{\prime}\right| \leq 2 m / T .\right.
$$

Similar to the proof Proposition 4.6, we see that $u \eta_{j} \in \mathbb{H}_{p, q, w, 0}^{\alpha, 2}\left(\left(s_{j-1}, s_{j+1}\right) \times \mathbb{R}^{d}\right)$ and satisfies

$$
-\partial_{t}^{\alpha}\left(u \eta_{j}\right)+a^{i j} D_{i j}\left(u \eta_{j}\right)+b^{i} D_{i}\left(u \eta_{j}\right)+c\left(u \eta_{j}\right)=f \eta_{j}+h_{j}
$$

in $\left(s_{j-1}, s_{j+1}\right) \times \mathbb{R}^{d}$, where $\partial_{t}^{\alpha}=\partial_{t} I_{s_{j-1}}^{1-\alpha}$ and

$$
h_{j}(t, x)=\frac{\alpha}{\Gamma(1-\alpha)} \int_{-\infty}^{t}(t-s)^{-\alpha-1}\left(\eta_{j}(s)-\eta_{j}(t)\right) u(s, x) d s .
$$

Since $\eta_{j}(t)=1$ for $t \geq s_{j}$,

$$
h_{j}(t, x)=\frac{\alpha}{\Gamma(1-\alpha)} \int_{-\infty}^{t}(t-s)^{-\alpha-1}\left(\eta_{j}(s)-\eta_{j}(t)\right) u(s, x) \chi_{s \leq s_{j}} d s
$$

for $t \in\left(s_{j-1}, s_{j+1}\right)$. In particular,

$$
h_{0}(t, x)=0,
$$

and, for $j=1,2, \ldots, m-1$,

$$
\begin{aligned}
& h_{j}(t, x)=\frac{\alpha}{\Gamma(1-\alpha)} \int_{0}^{t}(t-s)^{-\alpha-1}\left(\eta_{j}(s)-\eta_{j}(t)\right) u(s, x) \chi_{s \leq s_{j}} d s \\
& \leq \frac{2 m}{T} \frac{\alpha}{\Gamma(1-\alpha)} \int_{0}^{t}(t-s)^{-\alpha}|u(s, x)| \chi_{s \leq s_{j}} d s=\frac{2 m \alpha}{T} I_{0}^{1-\alpha}\left|u(\cdot, x) \chi \cdot \leq s_{j}\right|(t) .
\end{aligned}
$$

By Lemma 5.5 for $j=1,2, \ldots, m-1$,

$$
\begin{aligned}
& \left\|h_{j}\right\|_{p, q, w,\left(s_{j-1}, s_{j+1}\right)} \leq\left\|h_{j}\right\|_{p, q, w,\left(0, s_{j+1}\right)} \\
& \leq \frac{N}{T}\left(\frac{(j+1) T}{m}\right)^{1-\alpha}\left\|u(t, x) \chi_{t \leq s_{j}}\right\|_{p, q, w,\left(0, s_{j+1}\right)} \leq N T^{-\alpha}\|u\|_{p, q, w,\left(0, s_{j}\right)} .
\end{aligned}
$$

Here and in the sequel we denote $\|\cdot\|_{p, q, w,\left(\tau_{1}, \tau_{2}\right)}=\|\cdot\|_{L_{p, q, w}\left(\left(\tau_{1}, \tau_{2}\right) \times \mathbb{R}^{d}\right)}$. This estimate combined with Lemma 5.6 and Corollary 5.4 applied to (5.9) shows that

$$
\begin{aligned}
& \|u\|_{p, q, w,\left(s_{j}, s_{j+1}\right)} \leq\left\|u \eta_{j}\right\|_{p, q, w,\left(s_{j-1}, s_{j+1}\right)} \leq N\left(\frac{T}{m}\right)^{\alpha}\left\|\partial_{t}^{\alpha}\left(u \eta_{j}\right)\right\|_{p, q, w,\left(s_{j-1}, s_{j+1}\right)} \\
& \leq N_{0}\left(\frac{T}{m}\right)^{\alpha}\left\|f \eta_{j}\right\|_{p, q, w,\left(s_{j-1}, s_{j+1}\right)}+N_{0} m^{-\alpha}\|u\|_{p, q, w,\left(0, s_{j}\right)} \\
& \quad+N_{1}\left(\frac{T}{m}\right)^{\alpha}\|u\|_{p, q, w,\left(s_{j-1}, s_{j}\right)}+N_{1}\left(\frac{T}{m}\right)^{\alpha}\|u\|_{p, q, w,\left(s_{j}, s_{j+1}\right)},
\end{aligned}
$$


where $N_{0}=N_{0}\left(d, \delta, \alpha, p, q, K_{1}\right)$ and $N_{1}=N_{1}\left(d, \delta, \alpha, p, q, K_{1}, K_{0}, R_{0}\right)$. By taking a sufficiently large integer $m$ so that

$$
N_{1}\left(\frac{T}{m}\right)^{\alpha}<\frac{1}{2}
$$

we see that

$$
\|u\|_{p, q, w,\left(s_{j}, s_{j+1}\right)} \leq N\|f\|_{p, q, w,\left(0, s_{j+1}\right)}+N\|u\|_{p, q, w,\left(0, s_{j}\right)},
$$

where $N=N\left(d, \delta, \alpha, p, q, K_{1}, K_{0}, R_{0}, T\right)$ and $j=0,1, \ldots, m-1$. Upon noting that $\|u\|_{p, q, w,\left(0, s_{0}\right)}=0$ and using induction, we arrive at (5.8).

To prove the existence result, one can use the results in [6] for the unmixed case without weights and the argument in [5, Section 8], or alternatively use the a prior estimate proved above and the solvability of a simple equation presented in [14.

\section{REFERENCES}

[1] Jawerth, Björn. Weighted inequalities for maximal operators: linearization, localization and factorization. Amer. J. Math. 108(2):361-414, 1986.

[2] L. A. Caffarelli and I. Peral. On $W^{1, p}$ estimates for elliptic equations in divergence form. Comm. Pure Appl. Math., 51(1):1-21, 1998.

[3] Philippe Clément and Jan Prüss. Global existence for a semilinear parabolic Volterra equation. Math. Z. 209(1):17-26, 1992.

[4] Hongjie Dong and Doyoon Kim. On the $L_{p}$-solvability of higher order parabolic and elliptic systems with BMO coefficients. Arch. Ration. Mech. Anal., 199(3):889-941, 2011.

[5] Hongjie Dong and Doyoon Kim. On $L_{p}$-estimates for elliptic and parabolic equations with $A_{p}$ weights. Trans. Amer. Math. Soc., 370(7):5081-5130, 2018.

[6] Hongjie Dong and Doyoon Kim. $L_{p}$-estimates for time fractional parabolic equations with coefficients measurable in time. Adv. Math., 345:289-345, 2019.

[7] Hongjie Dong and Doyoon Kim. $L_{p}$-estimates for time fractional parabolic equations in divergence form with measurable coefficients. J. Funct. Anal., 278(3):108338, 66, 2020.

[8] N. V. Krylov and Hongjie Dong. Fully nonlinear elliptic and parabolic equations in weighted and mixed-norm sobolev spaces. Calc. Var. Partial Differential Equations, 58(4):Art. 145, $32 \mathrm{pp}, 2019$.

[9] Doyoon Kim. Parabolic equations with partially BMO coefficients and boundary value problems in Sobolev spaces with mixed norms. Potential Anal., 33(1):17-46, 2010.

[10] Ildoo Kim, Kyeong-Hun Kim, and Sungbin Lim. An $L_{q}\left(L_{p}\right)$-theory for the time fractional evolution equations with variable coefficients. Adv. Math., 306:123-176, 2017.

[11] N. V. Krylov. Parabolic and elliptic equations with VMO coefficients. Comm. Partial Differential Equations, 32(1-3):453-475, 2007.

[12] N. V. Krylov. Parabolic equations with VMO coefficients in Sobolev spaces with mixed norms. J. Funct. Anal., 250(2):521-558, 2007.

[13] N. V. Krylov. Lectures on elliptic and parabolic equations in Sobolev spaces, volume 96 of Graduate Studies in Mathematics. American Mathematical Society, Providence, RI, 2008.

[14] Daehan Park, Beom-Seok Han, Kyeong-Hun Kim. Weighted $L_{q}\left(L_{p}\right)$-estimate with muckenhoupt weights for the diffusion-wave equations with time-fractional derivatives. arXiv:1911.07437

[15] J. Prüss, Quasilinear parabolic Volterra equations in spaces of integrable functions, Semigroup Theory and Evolution Equations, Lecture Notes in Pure and Applied Mathematics, 135:401420, 1991.

[16] José L. Rubio de Francia. Factorization theory and $A_{p}$ weights. Amer. J. Math., 106(3):533547, 1984.

[17] Rico Zacher. Maximal regularity of type $L_{p}$ for abstract parabolic volterra equations. J. Evol. Equ., 5(1):79-103, 2005.

[18] Rico Zacher. Weak solutions of abstract evolutionary integro-differential equations in Hilbert spaces. Funkcial. Ekvac., 52(1):1-18, 2009. 
(H. Dong) Division of Applied Mathematics, Brown University, 182 George Street, Providence, RI 02912, USA

E-mail address: Hongjie_Dong@brown.edu

(D. Kim) Department of Mathematics, Korea University, 145 Anam-ro, Seongbuk-gu, Seoul, 02841, Republic of Korea

E-mail address: doyoon_kim@korea.ac.kr 